\title{
Cross-sectional dependence robust block bootstrap panel unit root tests
}

Citation for published version (APA):

Palm, F. C., Smeekes, S., \& Urbain, J. R. Y. J. (2011). Cross-sectional dependence robust block bootstrap panel unit root tests. Journal of Econometrics, 163(1), 85-104.

https://doi.org/10.1016/j.jeconom.2010.11.010

Document status and date:

Published: 01/07/2011

DOI:

10.1016/j.jeconom.2010.11.010

Document Version:

Publisher's PDF, also known as Version of record

Document license:

Taverne

Please check the document version of this publication:

- A submitted manuscript is the version of the article upon submission and before peer-review. There can be important differences between the submitted version and the official published version of record.

People interested in the research are advised to contact the author for the final version of the publication, or visit the DOI to the publisher's website.

- The final author version and the galley proof are versions of the publication after peer review.

- The final published version features the final layout of the paper including the volume, issue and page numbers.

Link to publication

\footnotetext{
General rights rights.

- You may freely distribute the URL identifying the publication in the public portal. please follow below link for the End User Agreement:

www.umlib.nl/taverne-license

Take down policy

If you believe that this document breaches copyright please contact us at:

repository@maastrichtuniversity.nl

providing details and we will investigate your claim.
}

Copyright and moral rights for the publications made accessible in the public portal are retained by the authors and/or other copyright owners and it is a condition of accessing publications that users recognise and abide by the legal requirements associated with these

- Users may download and print one copy of any publication from the public portal for the purpose of private study or research.

- You may not further distribute the material or use it for any profit-making activity or commercial gain

If the publication is distributed under the terms of Article $25 \mathrm{fa}$ of the Dutch Copyright Act, indicated by the "Taverne" license above, 


\title{
Cross-sectional dependence robust block bootstrap panel unit root tests
}

\author{
Franz C. Palm, Stephan Smeekes*, Jean-Pierre Urbain \\ Department of Quantitative Economics, Maastricht University, The Netherlands
}

\section{A R T I C L E I N F O}

\section{Article history:}

Available online 10 December 2010

\section{JEL classification:}

C15

$\mathrm{C} 23$

Keywords:

Block bootstrap

Panel unit root test

Cross-sectional dependence

\begin{abstract}
A B S T R A C T
In this paper we consider the issue of unit root testing in cross-sectionally dependent panels. We consider panels that may be characterized by various forms of cross-sectional dependence including (but not exclusive to) the popular common factor framework. We consider block bootstrap versions of the groupmean (Im et al., 2003) and the pooled (Levin et al., 2002) unit root coefficient DF tests for panel data, originally proposed for a setting of no cross-sectional dependence beyond a common time effect. The tests, suited for testing for unit roots in the observed data, can be easily implemented as no specification or estimation of the dependence structure is required. Asymptotic properties of the tests are derived for $T$ going to infinity and $N$ finite. Asymptotic validity of the bootstrap tests is established in very general settings, including the presence of common factors and cointegration across units. Properties under the alternative hypothesis are also considered. In a Monte Carlo simulation, the bootstrap tests are found to have rejection frequencies that are much closer to nominal size than the rejection frequencies for the corresponding asymptotic tests. The power properties of the bootstrap tests appear to be similar to those of the asymptotic tests.
\end{abstract}

(C) 2010 Elsevier B.V. All rights reserved.

\section{Introduction}

The use of panel data to test for unit roots and cointegration has become very popular recently. A major problem with tests for unit roots (and cointegration) in univariate time series is that they lack power for small sample sizes. Therefore one of the reasons people have turned to panel data, is to utilize the cross-sectional dimension to increase the power. Another reason to use panel data is that one might be interested in testing a joint unit root hypothesis for $N$ entities. The so-called first-generation panel unit root tests such as the tests proposed by Levin et al. (2002) and Im et al. (2003) are examples where the cross-sectional dimension is used to construct tests that have higher power than individual unit root tests. However, all the first-generation tests rely on independence along the cross-sectional dimension.

It was soon realized that cross-sectional independence is a highly unrealistic assumption for most settings encountered in practice, and it has been shown that the first-generation tests exhibit large size distortions in the presence of cross-sectional dependence (e.g. O'Connell, 1998). Therefore, the so-called secondgeneration panel unit root tests have been constructed to take

\footnotetext{
* Corresponding address: Department of Quantitative Economics, Maastricht University, P.O. Box 616, 6200 MD Maastricht, The Netherlands. Tel.: +31 43 3883856; fax: +31433884874.

E-mail addresses: F.Palm@maastrichtuniversity.nl (F.C. Palm), S.Smeekes@maastrichtuniversity.nl (S.Smeekes),

J.Urbain@maastrichtuniversity.nl (J.-P. Urbain).
}

the cross-sectional dependence into account in some way. These second-generation tests assume specific forms of the crosssectional dependence as their application depends on modelling the structure of the dependence. Most tests model the crosssectional dependence in the form of common factors, although the way the common factors are dealt with differs for each test. Examples of second-generation panel unit root tests are Bai and Ng (2004), Moon and Perron (2004), and Pesaran (2007). An extensive Monte Carlo comparison of these tests can be found in Gengenbach et al. (2010). Breitung and Das (2008) provide an analytical comparison of several first- and second-generation tests in the presence of factor structures.

While the second-generation panel unit root tests can deal with common factor structures and contemporaneous dependence, they cannot deal with other forms of cross-sectional dependence, with the exception of Pedroni et al. (2008). Of particular interest for practical applications are dynamic interrelationships (an example of which is Granger causality). Our goal in this paper is to present panel unit root tests that can deal not only with common factors, but also with a wide range of other plausible dynamic dependencies.

The tool we use to achieve this is the block bootstrap. Two very useful features of the block bootstrap are that one does not have to model the dependence (both temporal and cross-sectional) in order to apply it, and that it is valid to use under a wide range of possible data generating processes (DGPs). This makes it an appropriate tool to use in this setting with $N$ fixed, possibly large, and large $T$ asymptotics. 
Of course, the idea to use the bootstrap in cross-sectionally dependent panels is not new and has already been proposed by Maddala and Wu (1999), ${ }^{1}$ but so far no one has considered the theoretical properties of the block bootstrap in this setup. There are theoretical results available for other bootstrap and related resampling methods. Chang (2004) considers sieve bootstrap unit root tests, but the sieve bootstrap can only be applied in panels under restrictive assumptions on the cross-sectional dependence. Kapetanios (2008) proposes a bootstrap resampling scheme which resamples in the cross-sectional dimension instead of the usual time dimension, but this is based on cross-sectional independence. Choi and Chue (2007) consider subsampling, which does allow for more general dependence, but as the authors themselves state (p. 235) "Notwithstanding these nice features of the subsampling approach, depending on the nature of the problem at hand, other methods like bootstrapping may work better in finite samples".

Hence, the properties of the block bootstrap are still largely unknown in this setting, while in fact the block bootstrap is quite popular among practitioners. We try to fill this gap by providing theoretical results, mainly about asymptotic validity, of block bootstrap panel unit root tests. The block bootstrap method we consider here is the moving-blocks bootstrap (Künsch, 1989), and is an extension of the univariate bootstrap unit root test proposed by Paparoditis and Politis (2003). We will consider a very general DGP that can capture many different interesting and relevant forms of cross-sectional and time dependence.

Our results provide the theoretical justification, supported by Monte Carlo evidence, for the use of the proposed panel unit root tests in applications where one is interested in testing for a unit root in the observed data, and where cross-sectional dependence of possibly unknown form might be present in the data. The tests can be easily implemented, as they do not require the specification and estimation of the cross-sectional dependence structure. For example, it is not necessary to know the number of common factors, nor to estimate these factors. It is not even necessary to know whether common factors are present in the data at all.

The structure of this paper is as follows. Section 2 explains the model and assumptions. The test statistics and the construction of the bootstrap versions are discussed in Section 3. We establish the asymptotic validity of the bootstrap tests (for $T \rightarrow \infty$ and $N$ fixed) for various settings in Section 4 . Finite sample performance, including block length selection, is investigated in Section 5 . Section 6 concludes. All proofs and preliminary results are contained in the Appendix.

Finally, a word on notation. We use $|\cdot|$ to denote the Euclidean norm for vectors and matrices, i.e. $|v|=\left(v^{\prime} v\right)^{1 / 2}$ for a vector $v$ and $|M|=\left(\operatorname{tr} M^{\prime} M\right)^{1 / 2}$ for a matrix $M .\lfloor x\rfloor$ is the largest integer smaller than or equal to $x$. Convergence in distribution (probability) is denoted by $\stackrel{d}{\rightarrow}(\stackrel{p}{\rightarrow})$. Bootstrap quantities (conditional on the original sample) are indicated by appending a superscript $*$ to the standard notation.

\section{Cross-sectionally dependent panels}

Let us first describe the model that we use for panels with possible unit roots and that allows for various types of crosssectional and temporal dependence.

Let $y_{t}=\left(y_{1, t}, \ldots, y_{N, t}\right)^{\prime}(t=1, \ldots, T)$ be generated as

$y_{t}=\Lambda F_{t}+w_{t}$,

where $\Lambda=\left(\lambda_{1}, \ldots, \lambda_{N}\right)^{\prime}, F_{t}=\left(F_{1, t}, \ldots, F_{d, t}\right)^{\prime}$ and $w_{t}=$ $\left(w_{1, t}, \ldots, w_{N, t}\right)^{\prime}$. Hence, $F_{t}$ are common factors ( $d$ in total), $\Lambda$ are

\footnotetext{
1 Also see Fachin (2007) and Di Iorio and Fachin (2008) for some successful applications of the block bootstrap in testing for cointegration in panels.
}

the (non-random) factor loadings, and $w_{t}$ are the idiosyncratic components. Let $y_{0}=0$.

We let the factors and the idiosyncratic components be generated by

$F_{t}=\Phi F_{t-1}+f_{t}$,

$w_{t}=\Theta w_{t-1}+v_{t}$,

where $\Phi=\operatorname{diag}\left(\phi_{1}, \ldots, \phi_{d}\right)$ and $\Theta=\operatorname{diag}\left(\theta_{1}, \ldots, \theta_{N}\right)$.

Furthermore, we let $f_{t}$ and $v_{t}$ be constructed as

$\left[\begin{array}{l}v_{t} \\ f_{t}\end{array}\right]=\Psi(L) \varepsilon_{t}=\left[\begin{array}{ll}\Psi_{11}(L) & \Psi_{12}(L) \\ \Psi_{21}(L) & \Psi_{22}(L)\end{array}\right]\left[\begin{array}{l}\varepsilon_{v, t} \\ \varepsilon_{f, t}\end{array}\right]$,

where $\Psi(z)=\sum_{j=0}^{\infty} \Psi_{j} z^{j}\left(\Psi_{0}=I\right)$. We also partition $\Psi(z)$ as $\Psi(z)=\left(\Psi_{1}(z)^{\prime}, \Psi_{2}(z)^{\prime}\right)^{\prime}$ where $\Psi_{i}(z)=\left(\Psi_{i 1}(z), \Psi_{i 2}(z)\right), i=1,2$. We only need some mild conditions on $\Psi(z)$ and $\varepsilon_{t}$.

\section{Assumption 1.}

(i) $\operatorname{det}(\Psi(z)) \neq 0$ for all $\{z \in \mathbb{C}:|z|=1\}$ and $\sum_{j=0}^{\infty} j\left|\Psi_{j}\right|<\infty$.

(ii) $\varepsilon_{t}$ is i.i.d. with $\mathrm{E} \varepsilon_{t}=0, \mathrm{E} \varepsilon_{t} \varepsilon_{t}^{\prime}=\Sigma$ and $\mathrm{E}\left|\varepsilon_{t}\right|^{2+\epsilon}<\infty$ for some $\epsilon>0$.

Our null hypothesis is $H_{0}: y_{i, t}$ has a unit root for all $i=1, \ldots, N$. As in Bai and $\mathrm{Ng}$ (2004) and Breitung and Das (2008), we discern three different settings under which this can occur.

(A) $\theta_{i}=\phi_{j}=1$ for all $i=1, \ldots, N$ and $j=1, \ldots, d$ : both the common factors and the idiosyncratic components have a unit root. This is our first main setting.

(B) $\left|\theta_{i}\right|<1$ for all $i=1, \ldots, N, \phi_{j}=1$ for all $j=1, \ldots, d$ : the common factors have a unit root while the idiosyncratic components are stationary. In this setting the units are crosssectionally cointegrated. In accordance with most of the literature we shall call this cross-unit cointegration. We also discuss this case in detail. ${ }^{2}$

(C) $\theta_{i}=1$ for all $i=1, \ldots, N,\left|\phi_{j}\right|<1$ for all $j=1, \ldots, d$ : the common factors are stationary while the idiosyncratic components have a unit root. We shall not discuss this case in detail in Section 4 but its properties can easily be derived from the previous two cases.

Note that in this paper we are not interested in which of the three settings occurs, instead we simply want to test if $y_{i, t}$ has a unit root for all $i$.

We can discern different alternative hypotheses. ${ }^{3}$ The following two are of interest to us.

- $H_{1}^{a}: y_{i, t}$ is stationary for all $i=1, \ldots, N$. This implies that $\left|\theta_{i}\right|<1$ for all $i=1, \ldots, N$ and $\left|\phi_{j}\right|<1$ for all $j=1, \ldots, d$.

- $H_{1}^{b}: y_{i, t}$ is stationary for a portion of the units. This implies that $\left|\phi_{j}\right|<1$ for all $j=1, \ldots, d$; while $\left|\theta_{i}\right|<1$ for all $i \in \ell_{1}$ and $\theta_{i}=1$ for all $i \in \ell_{2}$, with $\ell_{1} \cup \ell_{2}=\{1, \ldots, N\}$ and $n_{1} / N=\kappa>0$, where $n_{1}$ is the number of elements of $\ell_{1} \cdot{ }^{4}$

\footnotetext{
2 We could also easily think of a setting in between settings (A) and (B), i.e. one where $\left|\theta_{i}\right|<1$ for all $i \in \ell_{1}$ and $\theta_{i}=1$ for all $i \in \ell_{2}$ (with $\ell_{1} \cup \ell_{2}=\{1, \ldots, N\}$ ). In other words, where part of the units are cointegrated and others are not. We will not analyze this setting in detail as it is basically contained in the analysis of settings (A) and (B).

3 Di Iorio and Fachin (2008) discuss several alternative hypotheses that are relevant when testing for the null of no panel cointegration. They also argue that the choice of the test statistic should depend on the alternative hypothesis. Their arguments are valid for the unit root setting as well.

4 In principle we could also let some of the factors be I(1) provided they have zero loadings on the units in $\ell_{1}$.
} 
In setting (B) we need an additional assumption on the factor loadings. In particular, we need to assume that for each unit at least one of the loadings on the factors is not equal to zero. If all loadings for one unit are equal to zero, this unit would be stationary and therefore the null hypothesis would be violated. This is formalized in Assumption 2.

Assumption 2. If setting (B) holds, then $\lambda_{i}^{\prime} \lambda_{i}>0$ for all $i=$ $1, \ldots, N$.

While this seems an additional and unverifiable assumption, this is not really the case. In fact, Assumption 2 is not really an assumption on the DGP as it is implied by our null hypothesis and in particular setting (B). One does therefore not have to verify whether this assumption holds when applying the test: under the null hypothesis it must hold by construction in setting (B), while in settings $(A)$ and $(C)$ it has no impact on the test.

Remark 1. Note that while the setting we adopt is fairly comparable to factor models such as those considered in Bai and $\mathrm{Ng}$ (2004) and Breitung and Das (2008), it is more general in several ways. First, it is common to assume $\Psi_{12}(z)=0, \Psi_{21}(z)=0$ and $\Sigma_{12}=0, \Sigma_{21}=0$ such that the factors are independent of the idiosyncratic components. There is however no need to do so in order to obtain our theoretical results, and therefore we will not make this assumption in general. Whenever this assumption is made, this will be explicitly mentioned.

Moreover, and more importantly, in most common factor models only weak dependence between the idiosyncratic components is allowed. We do not make this assumption; instead we allow for a wide array of possible dependencies between the idiosyncratic components, both through $\Sigma$ and $\Psi(z)$. Especially the lag polynomial allows for a wide range of dependencies, including all sorts of dynamic dependencies.

Therefore setting (A) is our main setting of interest, as simply setting $\lambda_{i}=0$ for all $i=1, \ldots, N$ results in a model without common factors, where the cross-sectional dependence is completely generated by $\Sigma$ and $\Psi(z)$. This setting is therefore the most general. We also analyze setting $(B)$ as it has generated a lot of attention in the literature (mainly due to Bai and $\mathrm{Ng}, 2004$ ), but it is in fact a very specialized setting that lacks the generality of setting $(A)$.

The DGP employed by Chang (2004) is also a special case of our DGP: in setting (A), one can obtain this DGP by setting $\lambda_{i}=0$ for all $i=1, \ldots, N$ and making $\Psi(L)$ diagonal and obey an invertibility condition.

Remark 2. One might wonder whether we can actually call $w_{t}$ idiosyncratic components given the degree of interdependence that we allow for, as $\Sigma_{11}$ and $\Psi_{11}(z)$ might be non-diagonal without restrictions beyond full rank and $\Psi_{12}(z)$ might be nonzero. In fact, one could even raise the question whether the distinction between factors and idiosyncratic components makes sense in our model with finite $N$. After all, the distinction between strong and weak dependence (that gives meaning to factors and idiosyncratic components) depends on the weak dependence disappearing as $N$ grows (see for example Chudik et al., 2009).

As a consequence of this the long-run covariance matrix will always have full rank in setting $(A)$ irrespective of whether factors are present. Therefore we could use a VMA model for $\Delta y_{t}$ instead. We could even use this for setting (B), for which we currently use the orders of integration of the factor structure and the idiosyncratic components to distinguish it from the other settings. To be precise, we could assume under the null hypothesis that

$\Delta y_{t}=\Psi(L) \varepsilon_{t}$.

Setting (B) would result from this by assuming that the rank of $\Psi(1)$ is equal to $d$. By the Beveridge-Nelson decomposition we would then have
$\Delta y_{t}=\Psi(1) \varepsilon_{t}+\tilde{\Psi}(L) \Delta \varepsilon_{t}$,

which, by writing $\Psi(1)=\Lambda \beta^{\prime}$ and letting $\Delta F_{t}=\beta^{\prime} \varepsilon_{t}$, could be rewritten as

$y_{t}=\Lambda F_{t}+\tilde{\Psi}(L) \varepsilon_{t}$.

The correspondence between this model and ours is obvious from this representation (this model could even be seen as a special case of ours with the differenced factors being white noise). The crucial point is that both this and our model allow us to deal with setting (B), where most existing tests fail. ${ }^{5}$

However, we stick to the factor structure model and terminology for two reasons. First, we want to stay close to the usual nonstationary panel models, such that our results remain comparable to the main strand of the literature. Also, from our model one can easily reach a "true" factor setup by imposing restrictions on the lag polynomial and the covariance matrix of the errors.

The second reason is for clarity. As mentioned above, we could do without the factor structure and model everything in terms of a VARMA for $y_{t}$. However, we would still need restrictions (such as on the rank) and the cases would therefore still need to be analyzed separately, as illustrated above. Therefore, little would be gained from this approach while we believe that the intuitive appeal of our setup and the interpretability would be lost.

\section{Bootstrap unit root tests in panels}

\subsection{Test statistics}

We will consider bootstrapping simplified versions of the Levin et al. (2002, LLC) and Im et al. (2003, IPS) test statistics. The first simplification is that we take the test statistics before corrections for mean and variance. The reason is that adding or multiplying the original test statistic and the bootstrap test statistic with the same number will obviously not have an effect on the performance of the tests. This is therefore a completely harmless simplification.

The second simplification is that we consider DF instead of ADF tests. Usually, the main reason to use ADF type of tests is to obtain asymptotically pivotal statistics. However, in the presence of complicated cross-sectional dependence it is often not possible to obtain asymptotically pivotal statistics anyway. There is therefore little reason (at least asymptotically) to use ADF instead of DF tests.

The third simplification is that we look at the DF coefficient test rather than the $t$-test. The main reason for this is that block bootstrapping naively studentized statistics may lead to serious problems in terms of accuracy of the tests as discussed for example in Section 3.1.2 of Härdle et al. (2003). As this is a second order problem, it does not lead to invalidity of the bootstrap, but it may cause the bootstrap to converge at a slower rate than the standard asymptotic approximation, although the evidence of this effect in finite samples is not always present. ${ }^{6}$

Given all these modifications, we prefer to call our test statistics "pooled" and "group-mean" instead of LLC and IPS, respectively. Note though that the essence of the LLC and IPS tests remains in our tests and that our methods can be trivially extended to the original LLC and IPS statistics if one so desires. ${ }^{7}$

\footnotetext{
5 Notable exceptions are Choi and Chue (2007) and Chang and Song (2009).

6 As pointed out by one of the referees, Gonçalves and Vogelsang (forthcoming) provide an asymptotic framework that can be used as an alternative to the framework based on Edgeworth expansions to explain their observation that naively studentized statistics actually perform better than corrected studentized statistics in finite samples.

7 Note that these tests could also be implemented when we have an unbalanced panel with different numbers of observations $T_{i}$ over time, provided of course the number of observations increases. The implementation of the block bootstrap in such a setting, while possible, becomes considerably more complicated.
} 
We define the pooled statistic as the Dickey-Fuller coefficient statistic from the pooled regression of $\Delta y_{i, t}$ on $y_{i, t-1}$. Then we can write the pooled statistic as

$\tau_{p}=T \frac{\sum_{i=1}^{N} \sum_{t=2}^{T} y_{i, t-1} \Delta y_{i, t}}{\sum_{i=1}^{N} \sum_{t=2}^{T} y_{i, t-1}^{2}}$.

We define our group-mean statistic as the average of the DF coefficient statistics from the individual regressions of $\Delta y_{i, t}$ on $y_{i, t-1}$ for each $i=1, \ldots, N$. We can then write the group-mean statistic as

$\tau_{g m}=\frac{1}{N} \sum_{i=1}^{N} T \frac{\sum_{t=2}^{T} y_{i, t-1} \Delta y_{i, t}}{\sum_{t=2}^{T} y_{i, t-1}^{2}}$.

\subsection{Bootstrap algorithm}

We employ the following block bootstrap algorithm, which is a multivariate extension of the algorithm proposed by Paparoditis and Politis (2003) to test for unit roots in univariate time series.

\section{Bootstrap Algorithm.}

1. Let

$\hat{u}_{i, t}=y_{i, t}-\hat{\rho}_{i} y_{i, t-1}-\frac{1}{T-1} \sum_{t=2}^{T}\left(y_{i, t}-\hat{\rho}_{i} y_{i, t-1}\right)$,

where

$$
\hat{\rho}_{i}=\frac{\sum_{t=2}^{T} y_{i, t-1} y_{i, t}}{\sum_{t=2}^{T} y_{i, t-1}^{2}} \text { for all } i=1, \ldots, N
$$

Let $\hat{u}_{t}=\left(\hat{u}_{1, t}, \ldots, \hat{u}_{N, t}\right)^{\prime}$.

2. Choose a block length $b$ (smaller than $T){ }^{8}$ Draw $i_{0}, \ldots, i_{k-1}$ i.i.d. from the uniform distribution on $\{1,2, \ldots, T-b\}$, where $k=\lfloor(T-2) / b\rfloor+1$ is the number of blocks.

3. Construct the bootstrap errors $u_{2}^{*}, \ldots, u_{T}^{*}$ as

$$
u_{t}^{*}=\hat{u}_{i_{m}+s},
$$

where $m=\lfloor(t-2) / b\rfloor$ and $s=t-m b-1$.

4. Let $y_{1}^{*}=y_{1}$ and construct $y_{t}^{*}$ for $t \geq 2$ recursively as

$$
y_{t}^{*}=y_{t-1}^{*}+u_{t}^{*} \text {. }
$$

5. Calculate the bootstrap versions of the group-mean and pooled statistics, that is, calculate

$$
\tau_{p}^{*}=T \frac{\sum_{i=1}^{N} \sum_{t=2}^{T} y_{i, t-1}^{*} \Delta y_{i, t}^{*}}{\sum_{i=1}^{N} \sum_{t=2}^{T} y_{i, t-1}^{* 2}},
$$

and

$$
\tau_{g m}^{*}=\frac{1}{N} \sum_{i=1}^{N} T \frac{\sum_{t=2}^{T} y_{i, t-1}^{*} \Delta y_{i, t}^{*}}{\sum_{t=2}^{T} y_{i, t-1}^{* 2}} .
$$

6. Repeat Steps 2-5 B times, obtaining bootstrap test statistics $\tau_{\kappa}^{* b}, b=1, \ldots, B, \kappa=p, g m$, and select the bootstrap critical value $c_{\alpha}^{*}$ as $c_{\alpha}^{*}=\max \left\{c: B^{-1} \sum_{b=1}^{B} I\left(\tau_{\kappa}^{* b}<c\right) \leq \alpha\right\}$, or equivalently as the $\alpha$-quantile of the ordered $\tau_{\kappa}^{* b}$ statistics. Reject the null of a unit root if $\tau_{\kappa}$, calculated from Eq. (4) if $\kappa=p$ or Eq. (5) if $\kappa=g m$, is smaller than $c_{\alpha}^{*}$, where $\alpha$ is the nominal level of the test.

Note that a crucial role in the analysis of our block bootstrap method will be played by the series

$u_{i, t}=y_{i, t}-\rho_{i} y_{i, t-1}$.

As in Paparoditis and Politis (2003), $\rho_{i}=1$ should correspond to a unit root in $y_{i, t}$, while $\rho_{i}<1$ should correspond to $y_{i, t}$ being stationary. Given our estimation of $\hat{\rho}_{i}$ in Step $1, \rho_{i}$ is implicitly defined as

$\rho_{i}=\lim _{t \rightarrow \infty} \frac{\mathrm{E}\left(y_{i, t-1} y_{i, t}\right)}{\mathrm{E}\left(y_{i, t-1}^{2}\right)}$

which fulfills these correspondences (Paparoditis and Politis, 2003, Example 2.1). ${ }^{9}$ Note that under $H_{0}$ we simply have that $u_{i, t}=$ $y_{i, t}-y_{i, t-1}$ for all $i=1, \ldots, N$ or in vector notation $u_{t}=\Delta y_{t}$.

We need that the estimator in Step 1 satisfies the properties $\hat{\rho}_{i}-\rho_{i}=O_{p}\left(T^{-1}\right)$ if $\rho_{i}=1$ and $\hat{\rho}_{i}-\rho_{i}=o_{p}(1)$ if $\rho_{i}<1$. Our OLS estimator satisfies these properties. ${ }^{10}$

We also need the following assumption on the block length.

Assumption 3. Let $b \rightarrow \infty$ and $b=o\left(T^{1 / 2}\right)$ as $T \rightarrow \infty$.

Remark 3. While we do not consider deterministic components, our tests can be modified to account for them in the same way as discussed by Levin et al. (2002) and Im et al. (2003). The crucial issue regarding the bootstrap tests is to implement exactly the same deterministic specification in the calculation of both the test statistic on the bootstrap sample and of the test statistic on the original sample. The only further modification of the bootstrap algorithm would be to include the appropriate deterministic components in Step 1 as well.

We will not discuss deterministic components in detail in this paper as it would detract from our main objective to deal with cross-sectional dependence. There is a vast literature on deterministic components and their impact. Part of the literature, for example on the local power of panel unit root tests in the case of incidental trends (Moon et al., 2007), depends on $N \rightarrow \infty$ and will therefore not apply here, although in finite samples these results will most likely have an impact on our tests as well.

We would like to stress that the bootstrap will not solve any issues that arise in the presence of deterministic components. Smeekes (2009) shows for univariate unit root testing that common methods of detrending can be applied to the bootstrap sample such that the asymptotic properties of the tests are correctly replicated as long as the method of detrending in the original sample and the bootstrap sample is the same. Moreover, by simulation it is found there that the properties of the bootstrap tests in finite samples closely follow the asymptotic tests they are based on. Therefore, deterministic components can be analyzed separately from the bootstrap.

\footnotetext{
9 Given our definition of $\rho_{i}$ it is clear that under stationarity we will always have $\left|\rho_{i}\right|<1$. Paparoditis and Politis (2003, Example 2.2) show that if one estimates and hence implicitly defines $\rho_{i}$ differently, for example through an ADF regression, it is not always the case that $\rho_{i}>-1$.

10 This is similar to the conditions needed by Paparoditis and Politis (2003, Remark 2.3). Although they always include an intercept in the regression, the rates of convergence are the same because we assume zero drift (see for example Davidson, 2000, Chapter 14). 
Remark 4. Unlike the methods considered by Moon and Perron (2004) and Pesaran (2007), which are essentially tests of the presence of a unit root in the idiosyncratic components as pointed out by Bai and $\mathrm{Ng}$ (2010), our methods are tests of the presence of a unit root in the observed data. Therefore in our setup there is no need to consider the properties of the common factors separately.

\section{Asymptotic properties}

In this section we will investigate the asymptotic properties of our (bootstrap) test statistics by letting $T$ go to infinity while keeping $N$ fixed. We study only $T$ asymptotics for two reasons. First, it is standard practice in studies on resampling methods; see for example Chang (2004) and Choi and Chue (2007). Second, it is very difficult to obtain meaningful theoretical results for infinite $N$ with our general model without making additional assumptions. However, as neither our bootstrap method nor our proofs of asymptotic validity depend on the finiteness of $N$, there is no reason to expect that asymptotic validity breaks down with joint $T$ and $N$ asymptotics.

\subsection{Asymptotic properties under the main null hypothesis}

In this section we investigate the validity of the bootstrap procedure proposed above in setting (A), i.e. where $\phi_{j}=1$ for all $j=1, \ldots, d$ and $\theta_{i}=1$ for all $i=1, \ldots, N$ or equivalently $\Phi=I_{d}$ and $\Theta=I_{N}$.

Note that under this null hypothesis we can write

$u_{t}=\Delta y_{t}=\Gamma^{\prime} x_{t}$,

where $\Gamma=\left(I_{N}, \Lambda\right)^{\prime}$, and

$x_{t}=\left(v_{t}^{\prime}, f_{t}^{\prime}\right)^{\prime}=\Psi(L) \varepsilon_{t}$.

\subsubsection{Asymptotic properties of the test statistics}

We start by presenting the asymptotic distributions for the original series. After all, the bootstrap test statistics should mimic these distributions. The first step is the invariance principle, or functional central limit theorem.

Lemma 1. Let $y_{t}$ be generated under $H_{0}$ setting (A) and let Assumption 1 hold. Then, as $T \rightarrow \infty$,

$S_{T}(r)=T^{-1 / 2} \sum_{t=1}^{\lfloor T r\rfloor} u_{t} \stackrel{d}{\rightarrow} B(r)$,

where $B(r)=\Gamma^{\prime} \Psi(1) \Sigma^{1 / 2} W(r)$ and $W(r)$ denotes an $(N+d)$ dimensional standard Brownian motion.

Next define

$\Omega=\lim _{T \rightarrow \infty} T^{-1} \mathrm{E}\left(\sum_{t=1}^{T} u_{t}\right)\left(\sum_{t=1}^{T} u_{t}\right)^{\prime}$ and

$\Omega_{0}=\lim _{T \rightarrow \infty} T^{-1} \sum_{t=1}^{T} \mathrm{E}\left(u_{t} u_{t}^{\prime}\right)$.

The limiting distributions now follow straightforwardly.

Theorem 1. Let $y_{t}$ be generated under $H_{0}$ setting (A) and let Assumption 1 hold. Then, as $T \rightarrow \infty$,

$\tau_{p} \stackrel{d}{\rightarrow} \frac{\sum_{i=1}^{N}\left[\int_{0}^{1} B_{i}(r) \mathrm{d} B_{i}(r)+\frac{1}{2}\left(\omega_{i}-\omega_{0, i}\right)\right]}{\sum_{i=1}^{N} \int_{0}^{1} B_{i}(r)^{2} \mathrm{~d} r}$ and

$\tau_{g m} \stackrel{d}{\rightarrow} \frac{1}{N} \sum_{i=1}^{N} \frac{\int_{0}^{1} B_{i}(r) \mathrm{d} B_{i}(r)+\frac{1}{2}\left(\omega_{i}-\omega_{0, i}\right)}{\int_{0}^{1} B_{i}(r)^{2} \mathrm{~d} r}$,

where $B_{i}(r)$ is the ith element of $B(r)=\Gamma^{\prime} \Psi(1) \Sigma^{1 / 2} W(r)$ and $\omega_{i}\left(\omega_{0, i}\right)$ is the $(i, i)$ th element of $\Omega\left(\Omega_{0}\right)$.

Remark 5. To see how the Brownian motion $B(r)$ depends on the idiosyncratic components and on the factors, consider the following. Let $B_{v}(r)=\Psi_{1}(1) \Sigma^{1 / 2} W(r)$ be the Brownian motion generated by the idiosyncratic components and $B_{f}(r)=\Psi_{2}(1) \Sigma^{1 / 2} W(r)$ the Brownian motion generated by the common factors. With this definition $B(r)=B_{v}(r)+\Lambda B_{f}(r)$. Note that if $\Psi_{12}(L)=\Psi_{21}(L)=0$ and $\Sigma_{12}=\Sigma_{21}=0$ we can write $B_{v}(r)=\Psi_{11}(1) \Sigma_{11}^{1 / 2} W_{1}(r)$ and $B_{f}(r)=\Psi_{22}(1) \Sigma_{22}^{1 / 2} W_{2}(r)$ where $W_{1}(r)$ is of dimension $N$ and $W_{2}(r)$ is of dimension $d$. For the $i$ th element of $B(r), B_{i}(r)$, we can then write $B_{i}(r)=B_{v, i}(r)+\lambda_{i}^{\prime} B_{f}(r)$. Note however that, given the points raised in Remark 2 , it is not possible to identify $B_{v}$ from $B_{f}$, and in that sense the distinction between the two is conceptual only.

\subsubsection{Asymptotic properties of the bootstrap test statistics}

Next we turn to the bootstrap test statistics. The first step is the bootstrap invariance principle.

Lemma 2. Let $y_{t}$ be generated under $H_{0}$ setting (A). Let Assumptions 1 and 3 hold. Then, as $T \rightarrow \infty$,

$S_{T}^{*}(r)=T^{-1 / 2} \sum_{t=1}^{\lfloor T r\rfloor} u_{t}^{*} \stackrel{d^{*}}{\rightarrow} B(r) \quad$ in probability.

Lemma 2 shows that the bootstrap partial sum process correctly mimics the original partial sum process. The limiting distributions of the bootstrap test statistics now follow as given below.

Theorem 2. Let $y_{t}$ be generated under $H_{0}$ setting (A). Let Assumptions 1 and 3 hold. Then, as $T \rightarrow \infty$,

$$
\tau_{p}^{*} \stackrel{d^{*}}{\rightarrow} \frac{\sum_{i=1}^{N}\left[\int_{0}^{1} B_{i}(r) \mathrm{d} B_{i}(r)+\frac{1}{2}\left(\omega_{i}-\omega_{0, i}\right)\right]}{\sum_{i=1}^{N} \int_{0}^{1} B_{i}(r)^{2} \mathrm{~d} r} \text { in probability }
$$

and

$\tau_{g m}^{*} \stackrel{d^{*}}{\rightarrow} \frac{1}{N} \sum_{i=1}^{N} \frac{\int_{0}^{1} B_{i}(r) \mathrm{d} B_{i}(r)+\frac{1}{2}\left(\omega_{i}-\omega_{0, i}\right)}{\int_{0}^{1} B_{i}(r)^{2} \mathrm{~d} r} \quad$ in probability.

Theorem 2 establishes the asymptotic validity of the proposed tests.

\subsection{Asymptotic properties of the tests under cross-unit cointegration}

In this section we look at setting (B), i.e. where $\Phi=I_{N}$ and $\theta_{i}<1$ for all $i=1, \ldots, N$ in (2). Note that in this case we may write

$u_{t}=\Delta y_{t}=\Lambda f_{t}+\Delta w_{t}=\Lambda f_{t}+(1-L)\left(I_{N}-\Theta L\right)^{-1} v_{t}$.

Now let

$\bar{\Psi}(z)=\left[\begin{array}{c}\left(I_{N}-\Theta L\right)^{-1} \Psi_{1}(z) \\ \Psi_{2}(z)\end{array}\right]$,

such that

$\left[\begin{array}{l}w_{t} \\ f_{t}\end{array}\right]=\bar{\Psi}(L) \varepsilon_{t}$.

Note that $\bar{\Psi}(z)$ satisfies Assumption 1 just as $\Psi(z)$. 


\subsubsection{Asymptotic properties of the test statistics}

We start again by presenting the invariance principle for the original series.

Lemma 3. Let $y_{t}$ be generated under $H_{0}$ setting (B). Let Assumptions 1 and 2 hold. Then, as $T \rightarrow \infty$,

$S_{T}(r)=T^{-1 / 2} \sum_{t=1}^{\lfloor T r\rfloor} u_{t} \stackrel{d}{\rightarrow} \bar{B}(r)$

where $\bar{B}(r)=\Lambda B_{f}(r)$ and $B_{f}(r)=\Psi_{2}(1) \Sigma^{1 / 2} W(r)$.

Note that the resulting Brownian motion $\bar{B}(r)$ has a reduced rank covariance matrix as it is only generated by the factors and not the idiosyncratic components.

Define

$\bar{\Omega}=\lim _{T \rightarrow \infty} T^{-1} \mathrm{E}\left(\sum_{t=1}^{T} u_{t}\right)\left(\sum_{t=1}^{T} u_{t}\right)^{\prime} \quad$ and

$\bar{\Omega}_{0}=\lim _{T \rightarrow \infty} T^{-1} \sum_{t=1}^{T} \mathrm{E}\left(u_{t} u_{t}^{\prime}\right)$.

Now we can derive the asymptotic distributions.

Theorem 3. Let $y_{t}$ be generated under $H_{0}$ setting (B). Let Assumptions 1 and 2 hold. Then, as $T \rightarrow \infty$,

$\tau_{p} \stackrel{d}{\rightarrow} \frac{\sum_{i=1}^{N}\left[\int_{0}^{1} \bar{B}_{i}(r) \mathrm{d} \bar{B}_{i}(r)+\frac{1}{2}\left(\bar{\omega}_{i}-\bar{\omega}_{0, i}\right)\right]}{\sum_{i=1}^{N} \int_{0}^{1} \bar{B}_{i}(r)^{2} \mathrm{~d} r}$

and

$\tau_{g m} \stackrel{d}{\rightarrow} \frac{1}{N} \sum_{i=1}^{N} \frac{\int_{0}^{1} \bar{B}_{i}(r) \mathrm{d} \bar{B}_{i}(r)+\frac{1}{2}\left(\bar{\omega}_{i}-\bar{\omega}_{0, i}\right)}{\int_{0}^{1} \bar{B}_{i}(r)^{2} \mathrm{~d} r}$,

where $\bar{B}_{i}(r)$ is the ith element of $\bar{B}(r)$ and $\bar{\omega}_{i}\left(\bar{\omega}_{0, i}\right)$ is the $(i, i)$ th element of $\bar{\Omega}\left(\bar{\Omega}_{0}\right)$.

\subsubsection{Asymptotic properties of the bootstrap test statistics}

Next we turn to the bootstrap series. Before presenting the bootstrap invariance principle, some discussion is in order.

As can be seen in Lemma 3, the Brownian motion generated by the partial sum process has reduced rank as it is only driven by the factors. In order to properly replicate the structure of the original series, the same should be true for the bootstrap partial sum process.

In the proof of Lemma 2 it is shown that the bootstrap series $u_{t}^{*}$ behaves approximately like $u_{i_{m}+s}$, ignoring centering for the moment. Summing over the variables within one block, we obtain

$\sum_{s=1}^{b} u_{i_{m}+s}=\sum_{s=1}^{b}\left(\Lambda f_{i_{m}+s}+\Delta w_{i_{m}+s}\right)=\sum_{s=1}^{b} \Lambda f_{i_{m}+s}+w_{i_{m}+b}-w_{i_{m}}$,

as all intermediate terms cancel against each other. This also happens in the partial sum of the original series and explains why only the factors contribute to the Brownian motion.

However, summing both over the blocks and within the blocks, we obtain

$$
\begin{aligned}
\sum_{m=0}^{\lfloor(k-1) r\rfloor} \sum_{s=1}^{b} u_{i_{m}+s} & =\sum_{m=0}^{\lfloor(k-1) r\rfloor}\left(\sum_{s=1}^{b}\left(\Lambda f_{i_{m}+s}+w_{i_{m}+b}-w_{i_{m}}\right)\right) \\
& =\sum_{m=0}^{\lfloor(k-1) r\rfloor} \sum_{s=1}^{b} \Lambda f_{i_{m}+s}+\sum_{m=0}^{\lfloor(k-1) r\rfloor}\left(w_{i_{m}+b}-w_{i_{m}}\right)
\end{aligned}
$$

where now the endpoints of the blocks do not cancel against each other as the blocks are randomly selected. The first term in this sum is the partial sum process of the factors, which generates the Brownian motion in Lemma 3 if we divide by $T^{1 / 2}$.

The second part is the partial sum process of the idiosyncratic components which generates an (unwanted) Brownian motion by dividing by $k^{1 / 2}$. As this rate is slower than $T^{1 / 2}$ by Assumption 3, the second part will vanish at rate $T^{1 / 2} / k^{1 / 2}$, so at rate $b^{1 / 2}$. Therefore, an increasing block length is crucial to make the second part vanish. In finite samples however one will always have a nonzero partial sum of the idiosyncratic components, although the magnitude will depend on both the sample size and the actual block length. Due to this, the covariance matrix of the resulting Brownian motion will almost always be of full rank in finite samples instead of reduced rank as in Lemma 3. It might therefore be expected that in this setting the block bootstrap might not work optimally in finite samples, although it is also clear that large block lengths should improve the performance of the tests in this case.

Remark 6. The result under the null hypothesis of cross-unit cointegration is closely related to the result obtained by Paparoditis and Politis (2003, Lemma 8.5) for the difference-based block bootstrap (DBB) under the alternative. In both cases one bootstraps an over-differenced series. However, as the result for the DBB is under the alternative hypothesis, the different bootstrap stochastic order leads to serious (power) problems, whereas in our setting, under the null hypothesis, it is what preserves the validity of the bootstrap tests. The result described above is formalized in Lemma A.9 in the Appendix.

Given the discussion above, it is clear that the bootstrap validity is preserved in this setting, giving rise to the following bootstrap invariance principle.

Lemma 4. Let $y_{t}$ be generated under $H_{0}$ setting (B). Let Assumptions 1-3 hold. Then, as $T \rightarrow \infty$,

$S_{T}^{*}(r)=T^{-1 / 2} \sum_{t=1}^{\lfloor T r\rfloor} u_{t}^{*} \stackrel{d^{*}}{\rightarrow} \bar{B}(r) \quad$ in probability.

Finally we derive the limiting distributions of the test statistics, again establishing the asymptotic validity of the bootstrap tests.

Theorem 4. Let $y_{t}$ be generated under $H_{0}$ setting (B). Let Assumptions 1-3 hold. Then, as $T \rightarrow \infty$,

$\tau_{p}^{*} \stackrel{d^{*}}{\rightarrow} \frac{\sum_{i=1}^{N}\left[\int_{0}^{1} \bar{B}_{i}(r) \mathrm{d} \bar{B}_{i}(r)+\frac{1}{2}\left(\bar{\omega}_{i}-\bar{\omega}_{0, i}\right)\right]}{\sum_{i=1}^{N} \int_{0}^{1} \bar{B}_{i}(r)^{2} \mathrm{~d} r}$ in probability

and

$\tau_{g m}^{*} \stackrel{d^{*}}{\rightarrow} \frac{1}{N} \sum_{i=1}^{N} \frac{\int_{0}^{1} \bar{B}_{i}(r) \mathrm{d} \bar{B}_{i}(r)+\frac{1}{2}\left(\bar{\omega}_{i}-\bar{\omega}_{0, i}\right)}{\int_{0}^{1} \bar{B}_{i}(r)^{2} \mathrm{~d} r} \quad$ in probability.

\subsection{Asymptotic properties under the alternative hypothesis}

Let us start by considering the alternative $H_{1}^{a}$ (stationarity for all $y_{i, t}$ ).

Let us define

$y_{t}=\Lambda\left(I_{d}-\Phi L\right)^{-1} f_{t}+\left(I_{N}-\Theta L\right)^{-1} v_{t}=\Gamma^{\prime} \Psi^{+}(L) \varepsilon_{t}$,

where

$\Psi^{+}(L)=\left[\begin{array}{c}\left(I_{N}-\Theta L\right)^{-1} \Psi_{1}(L) \\ \left(I_{d}-\Phi L\right)^{-1} \Psi_{2}(L)\end{array}\right]$.

Note that the lag polynomial $\Psi^{+}(z)$ meets the conditions in Assumption 1. 
We start by describing the asymptotic properties of our test statistics.

Lemma 5. Let $y_{t}$ be generated under $H_{1}^{a}$. Let Assumption 1 hold. Then, as $T \rightarrow \infty$,

$T^{-1} \tau_{p} \stackrel{p}{\rightarrow} \frac{\sum_{i=1}^{N}\left(\gamma_{i}(1)-\gamma_{i}(0)\right)}{\sum_{i=1}^{N} \gamma_{i}(0)}$

and

$T^{-1} \tau_{g m} \stackrel{p}{\rightarrow} N^{-1} \sum_{i=1}^{N} \frac{\gamma_{i}(1)-\gamma_{i}(0)}{\gamma_{i}(0)}$,

where $\gamma_{i}(j)=\mathrm{E}\left(y_{i, t-j} y_{i, t}\right)$.

Lemma 5 shows that both test statistics diverge to $-\infty$ under $H_{1}^{a}$ as $\gamma_{i}(1)<\gamma_{i}(0)$ for all $i=1, \ldots, N$. This is a necessary, but not a sufficient step in showing consistency of the bootstrap tests. The second step that is needed is to show that the bootstrap tests, and correspondingly the bootstrap critical values, do not diverge under $H_{1}^{a}$.

To this end, let $P=\operatorname{diag}\left(\rho_{1}, \ldots, \rho_{N}\right)$ and consequently $u_{t}=$ $\left(I_{N}-P L\right) y_{t}$. Then

$u_{t}=\left(I_{N}-P L\right) \Gamma^{\prime} \Psi^{+}(L) \varepsilon_{t}=\Psi^{++}(L) \varepsilon_{t}$,

where $\Psi^{++}(L)=\left(I_{N}-P L\right) \Gamma^{\prime} \Psi^{+}$. Note that the summability condition from Assumption 1 still holds for this lag polynomial. Therefore we can give the following theorem.

Theorem 5. Let $y_{t}$ be generated under $H_{1}^{a}$. Let Assumptions 1 and 3 hold. Then, as $T \rightarrow \infty$,

$\tau_{p}^{*} \stackrel{d^{*}}{\rightarrow} \frac{\sum_{i=1}^{N}\left[\int_{0}^{1} B_{i}^{+}(r) \mathrm{d} B_{i}^{+}(r)+\frac{1}{2}\left(\omega_{i}^{+}-\omega_{0, i}^{+}\right)\right]}{\sum_{i=1}^{N} \int_{0}^{1} B_{i}^{+}(r)^{2} \mathrm{~d} r}$ in probability,

and

$\tau_{g m}^{*} \stackrel{d^{*}}{\rightarrow} \frac{1}{N} \sum_{i=1}^{N} \frac{\int_{0}^{1} B_{i}^{+}(r) \mathrm{d} B_{i}^{+}(r)+\frac{1}{2}\left(\omega_{i}^{+}-\omega_{0, i}^{+}\right)}{\int_{0}^{1} B_{i}^{+}(r)^{2} \mathrm{~d} r}$ in probability,

where $B_{i}^{+}(r)$ is the ith element of $B^{+}(r)=\Psi^{++}(1) \Sigma^{1 / 2} W(r)$ and $\omega_{i}^{+}$and $\omega_{0, i}^{+}$are the $(i, i)$ th elements of $\Omega^{+}=\Psi^{++}(1) \Sigma \Psi^{++}(1)^{\prime}$ and $\Omega_{0}^{+}=\sum_{j=0}^{\infty} \Psi_{j}^{++} \Sigma \Psi_{j}^{++\prime}$, respectively.

Note that Lemma 5 and Theorem 5 jointly establish the consistency of our tests.

Let us now consider $H_{1}^{b}$. Again we first look at the properties of the test statistics. Let us first without loss of generality assume that the first $n_{1}$ units are $I(0)$, while the rest is $I(1)$. Hence, $\rho_{i}<1$ for $i=1, \ldots, n_{1}$ and $\rho_{i}=1$ for $i=n_{1}+1, \ldots, N$. We can then define $u_{t}=y_{t}-P y_{t-1}$, where parts of the $\rho_{i}$ are equal to one and the rest is smaller than one. We may then write that

$u_{t}=\Psi^{\#}(L) \varepsilon_{t}$,

where the values for $\Psi^{\#}(L)$ for the $I(1)$ components are determined as in the analysis under the null, and for the $I(0)$ components as in the analysis above. The summability condition will clearly still hold for $\Psi^{\#}(L)$. The limit behavior of the test statistics is then given by the following lemma.

Lemma 6. Let $y_{t}$ be generated under $H_{1}^{b}$. Let Assumption 1 hold. Then, as $T \rightarrow \infty$,

$$
\tau_{p} \stackrel{d}{\rightarrow} \frac{\sum_{i=1}^{n_{1}}\left(\gamma_{i}(1)-\gamma_{i}(0)\right)+\sum_{i=n_{1}+1}^{N}\left(\int_{0}^{1} B_{i}^{\#}(r) \mathrm{d} B_{i}^{\#}(r)+\frac{1}{2}\left(\omega_{i}^{\#}-\omega_{0, i}^{\#}\right)\right)}{\sum_{i=n_{1}+1}^{N} \int_{0}^{1} B_{i}^{\#}(r)^{2} \mathrm{~d} r}
$$

and

$T^{-1} \tau_{g m} \stackrel{p}{\rightarrow} N^{-1} \sum_{i=1}^{n_{1}} \frac{\gamma_{i}(1)-\gamma_{i}(0)}{\gamma_{i}(0)}$,

where $\gamma_{i}(j)=\mathrm{E}\left(y_{i, t-j} y_{i, t}\right), B_{i}^{\#}(r)$ is the ith element of $B^{\#}(r)=$ $\Psi^{\#}(1) \Sigma^{1 / 2} W(r)$ and $\omega_{i}^{\#}$ and $\omega_{0, i}^{\#}$ are the $(i, i)$ th elements of $\Omega^{\#}=$ $\Psi^{\#}(1) \Sigma \Psi^{\#}(1)^{\prime}$ and $\Omega_{0}^{\#}=\sum_{j=0}^{\infty} \Psi_{j}^{\#} \Sigma \Psi_{j}^{\# \prime}$, respectively.

We see that the group-mean statistic diverges to $-\infty$ as it should. The pooled statistic does not diverge however, which means that it is not consistent against this alternative. This is in fact not surprising, given that the pooled test is designed as a large $\mathrm{N}$ test for homogeneous alternatives (also see Remark 8). The reason for the inconsistency is that in the denominator the stationary units vanish (as they should) as $T \rightarrow \infty$, but the nonstationary units remain.

Let us turn to the bootstrap series. Given our expression of $u_{t}$ above, we can simply combine the proofs for the unit root and stationary series and directly state the limiting distributions as a corollary.

Corollary 1. Let $y_{t}$ be generated under $H_{1}^{b}$. Let Assumptions 1 and 3 hold. Then, as $T \rightarrow \infty$,

$$
\tau_{p}^{*} \stackrel{d^{*}}{\rightarrow} \frac{\sum_{i=1}^{N} \int_{0}^{1}\left[B_{i}^{\#}(r) \mathrm{d} B_{i}^{\#}(r)+\frac{1}{2}\left(\omega_{i}^{\#}-\omega_{0, i}^{\#}\right)\right]}{\sum_{i=1}^{N} \int_{0}^{1} B_{i}^{\#}(r)^{2} \mathrm{~d} r} \text { in probability, }
$$

and

$\tau_{g m}^{*} \stackrel{d^{*}}{\rightarrow} \frac{1}{N} \sum_{i=1}^{N} \frac{\int_{0}^{1} B_{i}^{\#}(r) \mathrm{d} B_{i}^{\#}(r)+\frac{1}{2}\left(\omega_{i}^{\#}-\omega_{0, i}^{\#}\right)}{\int_{0}^{1} B_{i}^{\#}(r)^{2} \mathrm{~d} r}$ in probability.

Note that Lemma 6 and Corollary 1 jointly establish the consistency of the bootstrap group-mean test. Also note that the inconsistency of the pooled test does not depend on the bootstrap distribution, but purely on the original test statistic.

Remark 7. It might seem that our bootstrap method does not correctly reproduce the asymptotic null distribution if the alternative is true as the nuisance parameters are different from those appearing in Theorem 2 for example, but this is not so straightforward. It all depends on how the alternative is formulated in relation to the null. Had we formulated our alternative as $y_{t}=P y_{t-1}+u_{t}$ where $u_{t}=\Gamma^{\prime} \Psi(L) \varepsilon_{t}$, the nuisance parameters would have been the same. The key to understanding this is that the process under the null corresponding to the process in (1) and (2) with $\Phi$ and $\Theta$ implying stationarity is not necessarily the same process with $\Phi=I_{d}$ and $\Theta=I_{N}$.

Remark 8. A few qualifications are in order regarding the inconsistency of the pooled test. First, the actual location of the pooled test can be seen to depend on both the proportion of stationary units (through $n_{1}$ in the sums) and the distance from the null (through the quantity $\gamma_{i}(1)-\gamma_{i}(0)$ ). If either becomes larger, the statistic will become more negative. Second, if $T$ increases, the denominator will become smaller as the sum over the stationary units disappears (the $b_{i T}$ part in the proof). Hence the test statistic will grow larger with increasing $T$, but the denominator will not go to zero as the nonstationary part does not vanish. Both factors imply that the actual power of the test can still be non-trivial and even reach 1 . 


\section{Small sample performance}

In this section we will investigate the small sample properties of our tests using Monte Carlo simulations. First we perform a simulation study to investigate the properties of our tests while fixing the block length to be a function of $T$ only. Next we will perform a separate and smaller simulation study to investigate the selection of block lengths.

\subsection{Monte Carlo design}

We consider the following DGP for the simulation study.

$y_{t}=\Lambda F_{t}+w_{t}$,

where $F_{t}$ is a single (scalar) factor and

$F_{t}=\phi F_{t-1}+f_{t}$,

$w_{i, t}=\theta_{i} w_{i, t-1}+v_{i, t}$.

Furthermore,

$v_{t}=A_{1} v_{t-1}+\varepsilon_{1, t}+B_{1} \varepsilon_{1, t-1}$,

$f_{t}=\alpha_{2} f_{t-1}+\varepsilon_{2, t}+\beta_{2} \varepsilon_{2, t-1}$,

where $\varepsilon_{2, t} \sim N(0,1)$ and

$\varepsilon_{1, t} \sim N(0, \Sigma)$,

where $\Sigma$ is generated as in Chang (2004):

1. Generate an $N \times N$ matrix $U \sim U[0,1]$. Construct $H=$ $U\left(U^{\prime} U\right)^{-1 / 2}$.

2. Generate $N$ eigenvalues $\zeta_{1}, \ldots, \zeta_{N}$ with $\zeta_{1}=r, \zeta_{N}=1$ and $\zeta_{i} \sim U[r, 1]$ for $i=2, \ldots, N-1$.

3. Let $Z=\operatorname{diag}\left(\zeta_{1}, \ldots, \zeta_{N}\right)$. Then let $\Sigma=H Z H^{\prime}$.

We consider both $r=1$ (no cross-sectional dependence) and $r=0.1$.

We consider five settings regarding the parameters in equations (23) and (25) in accordance with Gengenbach et al. (2010).

I No common factor, unit root for all idiosyncratic components: $\lambda_{i}=0, \theta_{i}=1$ for all $i=1, \ldots, N$.

II Unit root in common factor and idiosyncratic components: $\phi=$ $1, \theta_{i}=1$ for all $i=1, \ldots, N$ and $\lambda_{i} \sim U[-1,3]$.

III Unit root in common factor, stationary idiosyncratic components: $\phi=1, \theta_{i} \sim U[0.8,1]$ and $\lambda_{i} \sim U[-1,3]$. This is the setting of cross-unit cointegration.

IV No common factor, stationary idiosyncratic component: $\theta_{i} \sim$ $U[0.8,1]$ and $\lambda_{i}=0$ for all $i=1, \ldots, N$. This is under the alternative hypothesis. ${ }^{11}$

V Stationary common factor and idiosyncratic component: $\phi=$ $0.95, \theta_{i} \sim U[0.8,1]$ and $\lambda_{i} \sim U[-1,3]$. This is also under the alternative hypothesis. $B_{1}$ :

We consider two different options for the parameters $A_{1}$ and

1. No dynamic dependence: $A_{1}=B_{1}=0$.

2. Dynamic autoregressive moving-average cross-sectional dependence: $A_{1}$ and $B_{1}$ are non-diagonal.

We let $A_{1}=\Xi$, where

\footnotetext{
11 The reported power estimates are not size adjusted. We give raw power as we believe this is empirically more relevant than the usual size-corrected power; also see Horowitz and Savin (2000) for a discussion on the empirical relevance of the usual size-corrected powers in Monte Carlo simulations.
}

$$
\Xi=\left[\begin{array}{ccccc}
\xi_{1} & \xi_{1} \eta_{1} & \xi_{1} \eta_{1}^{2} & \ldots & \xi_{1} \eta_{1}^{N-1} \\
\xi_{2} \eta_{2} & \xi_{2} & \xi_{2} \eta_{2} & \ldots & \xi_{2} \eta_{2}^{N-2} \\
\vdots & & \ddots & & \vdots \\
\xi_{N} \eta_{N}^{N-1} & \ldots & \xi_{N} \eta_{N}^{2} & \xi_{N} \eta_{N} & \xi_{N}
\end{array}\right]
$$

where $\xi_{i}, \eta_{i} \sim U[-0.5,0.5]$. To ensure stationarity and invertibility we impose that $\operatorname{det}\left(I_{N}-A_{1} z\right) \neq 0$ for $\{z \in \mathbb{C}$ : $|z| \leq 1.2\}$.

Furthermore, we let $B_{1}=\Omega$. We construct $\Omega$ in much the same way as $\Sigma$. Let $M=H L H^{\prime}$ where $H=U\left(U^{\prime} U\right)^{-1 / 2}$, with $U$ an $N \times 1$-vector of $U[0,1]$-variables, and define $L$ as a diagonal matrix with on the diagonal $\ell_{1}, \ldots, \ell_{N}$ where $\ell_{1}=0.1, \ell_{N}=1$ and $\ell_{2}, \ldots, \ell_{N-1} \sim U[0.1,1]$. We then let $\Omega=2 M-I_{N}$. By generating $\Omega$ this way we assure that $I_{N}+\Omega$ is of full rank. Note that invertibility is not guaranteed (on purpose).

The parameters of the common factor in (25), $\alpha_{2}$ and $\beta_{2}$, are taken in accordance with the setting for the idiosyncratic components, so if the dependence for the idiosyncratic components is of the ARMA type, then the same will hold for the common factor. Note that for both $\Sigma$ and the $\Psi(1)$ matrix derived from $A_{1}$ and $B_{1}$ the eigenvalues are bounded if $N \rightarrow \infty$; as such these parameters can be regarded as weak dependence parameters.

For all combinations of the parameters described above we consider all combinations of $T=25,50,100$ and $N=5,25,50$. As several parameters in our DGP are chosen randomly, we repeat the simulations for each setting ten times, and store the mean, median, minimum and maximum. We only report results for the mean here. The mean is representative as in general there is little dispersion between the simulation results. The other results are available upon request. The results are based on 2000 simulations and the Warp-Speed bootstrap (Giacomini et al., 2007) is used to obtain estimates for the rejection frequencies of the bootstrap tests. ${ }^{12}$ The nominal level is 0.05 . The approximate confidence interval around 0.05 with 2000 simulations is $(0.042,0.058){ }^{13}$

In our simulation study we consider the LLC and IPS tests (with lag lengths selected by BIC), denoted by $\tau_{l l c}$ and $\tau_{\text {ips }}$ respectively, and the bootstrap pooled and group-mean tests, denoted by $\tau_{p}$ and $\tau_{g m}$. We also consider a bootstrap test based on the median of the individual test statistics, denoted by $\tau_{\text {med }}$. This test might be more robust to outlying units than the test based on the mean (also see the discussion in Di Iorio and Fachin, 2008). While we do not consider this test explicitly in our theoretical analysis as the median presents difficulties for asymptotic analysis, it is clear that a median-based test will be valid as well as we can show that the joint bootstrap distribution of the individual DF statistics is asymptotically valid. Block lengths of the bootstrap tests were taken as $b=1.75 T^{1 / 3}$, which amounts to blocks of length 6,7 and 9 for sample sizes 25,50 and 100 respectively, which is within the range usually considered in the literature. We return to the issue of block length selection in Section 5.3.

\subsection{Monte Carlo results}

Table 1 presents results for the setting without common factors. It can be noted in general that the asymptotic tests have poor size for $T=25$, which is mainly caused by the performance of the BIC, as this tends to select too large lag lengths for $T=25 .{ }^{14}$ From

\footnotetext{
12 The Warp-Speed bootstrap greatly reduces the computational cost of performing the simulations by only drawing one bootstrap replication for each simulation. Giacomini et al. (2007) show that under quite general conditions the Warp-Speed bootstrap is capable of calibrating the finite sample coverage of bootstrap confidence intervals if the bootstrap is asymptotically valid (which we show in this paper). Because of the close relation between confidence intervals and hypothesis testing the method should work properly in our setting as well.

13 This interval only takes into account the randomness of the simulations, not of the Warp-Speed bootstrap, nor takes into account that we take averages of 10 runs. 14 A similar result was obtained by Hlouskova and Wagner (2006).
} 
Table 1

Size properties without common factors (setting I).

\begin{tabular}{|c|c|c|c|c|c|c|c|c|}
\hline$A_{1}, B_{1}$ & $\Sigma$ & $T$ & $N$ & $\tau_{l l c}$ & $\tau_{p}$ & $\tau_{i p s}$ & $\tau_{g m}$ & $\tau_{\text {med }}$ \\
\hline \multicolumn{9}{|c|}{ Panel A: No short-run dependence } \\
\hline$A_{1}=0$ & $r=1$ & 25 & 5 & 0.140 & 0.024 & 0.141 & 0.020 & 0.025 \\
\hline \multirow[t]{8}{*}{$B_{1}=0$} & & 25 & 25 & 0.211 & 0.001 & 0.183 & 0.005 & 0.009 \\
\hline & & 25 & 50 & 0.260 & 0.000 & 0.207 & 0.001 & 0.002 \\
\hline & & 50 & 5 & 0.076 & 0.031 & 0.051 & 0.024 & 0.033 \\
\hline & & 50 & 25 & 0.063 & 0.004 & 0.060 & 0.011 & 0.014 \\
\hline & & 50 & 50 & 0.055 & 0.000 & 0.056 & 0.003 & 0.004 \\
\hline & & 100 & 5 & 0.077 & 0.032 & 0.049 & 0.032 & 0.035 \\
\hline & & 100 & 25 & 0.062 & 0.009 & 0.051 & 0.014 & 0.020 \\
\hline & & 100 & 50 & 0.056 & 0.001 & 0.051 & 0.005 & 0.010 \\
\hline \multicolumn{9}{|c|}{ Panel B: Contemporaneous, but no dynamic dependence } \\
\hline$A_{1}=0$ & $r=0.1$ & 25 & 5 & 0.151 & 0.026 & 0.159 & 0.022 & 0.031 \\
\hline \multirow[t]{8}{*}{$B_{1}=0$} & & 25 & 25 & 0.200 & 0.003 & 0.197 & 0.006 & 0.010 \\
\hline & & 25 & 50 & 0.236 & 0.000 & 0.215 & 0.001 & 0.002 \\
\hline & & 50 & 5 & 0.111 & 0.033 & 0.070 & 0.028 & 0.037 \\
\hline & & 50 & 25 & 0.077 & 0.006 & 0.072 & 0.009 & 0.017 \\
\hline & & 50 & 50 & 0.067 & 0.001 & 0.069 & 0.004 & 0.005 \\
\hline & & 100 & 5 & 0.113 & 0.040 & 0.066 & 0.031 & 0.039 \\
\hline & & 100 & 25 & 0.084 & 0.013 & 0.064 & 0.015 & 0.021 \\
\hline & & 100 & 50 & 0.073 & 0.003 & 0.067 & 0.007 & 0.012 \\
\hline \multicolumn{9}{|c|}{ Panel C: No contemporaneous, but dynamic dependence } \\
\hline$A_{1}=\Xi$ & $r=1$ & 25 & 5 & 0.215 & 0.054 & 0.207 & 0.082 & 0.055 \\
\hline \multirow[t]{8}{*}{$B_{1}=\Omega$} & & 25 & 25 & 0.235 & 0.004 & 0.198 & 0.032 & 0.016 \\
\hline & & 25 & 50 & 0.280 & 0.000 & 0.237 & 0.010 & 0.004 \\
\hline & & 50 & 5 & 0.154 & 0.052 & 0.123 & 0.097 & 0.054 \\
\hline & & 50 & 25 & 0.113 & 0.008 & 0.097 & 0.032 & 0.020 \\
\hline & & 50 & 50 & 0.109 & 0.001 & 0.106 & 0.023 & 0.010 \\
\hline & & 100 & 5 & 0.152 & 0.067 & 0.110 & 0.099 & 0.064 \\
\hline & & 100 & 25 & 0.130 & 0.013 & 0.108 & 0.028 & 0.023 \\
\hline & & 100 & 50 & 0.117 & 0.004 & 0.096 & 0.026 & 0.015 \\
\hline \multicolumn{9}{|c|}{ Panel D: Contemporaneous and dynamic dependence } \\
\hline$A_{1}=\Xi$ & $r=0.1$ & 25 & 5 & 0.222 & 0.056 & 0.212 & 0.063 & 0.051 \\
\hline \multirow[t]{8}{*}{$B_{1}=\Omega$} & & 25 & 25 & 0.252 & 0.003 & 0.238 & 0.020 & 0.012 \\
\hline & & 25 & 50 & 0.265 & 0.000 & 0.214 & 0.007 & 0.003 \\
\hline & & 50 & 5 & 0.197 & 0.049 & 0.146 & 0.059 & 0.046 \\
\hline & & 50 & 25 & 0.144 & 0.011 & 0.131 & 0.052 & 0.030 \\
\hline & & 50 & 50 & 0.127 & 0.001 & 0.119 & 0.018 & 0.008 \\
\hline & & 100 & 5 & 0.187 & 0.055 & 0.129 & 0.094 & 0.054 \\
\hline & & 100 & 25 & 0.158 & 0.012 & 0.129 & 0.029 & 0.023 \\
\hline & & 100 & 50 & 0.143 & 0.004 & 0.119 & 0.027 & 0.015 \\
\hline
\end{tabular}

$A_{1}, B_{1}$ and $\Sigma$ with smallest eigenvalue $r$ are defined in Section 5.1.

$\tau_{l l c}=$ LLC test; $\tau_{p}=$ pooled bootstrap test; $\tau_{i p s}=$ IPS test; $\tau_{g m}=$ group-mean bootstrap test; $\tau_{m e d}=$ median bootstrap test.

$T=50$ on this does not happen anymore. Panel A presents results for the setting without any dependence (both temporal and crosssectional). It can be seen that the asymptotic tests have good size properties for $T=50$ and $T=100$, while the bootstrap tests are undersized increasing in $N$. Panel B lists results for the setting where there is only contemporaneous correlation. The asymptotic tests have slight positive size distortions here, while the bootstrap tests are somewhat undersized. Panel $\mathrm{C}$ and $\mathrm{D}$ give results for the model with autoregressive moving-average errors. It is clear here that the asymptotic tests are quite oversized, while the bootstrap tests perform well although there is some undersize increasing with $N$. There is little difference between the three bootstrap tests.

Table 2 present the results for the model with a nonstationary common factor and nonstationary idiosyncratic components. For all three settings considered the table shows that the bootstrap tests have good size properties, while the asymptotic tests have large size distortions increasing with $N$. The bootstrap tests again perform very similarly.

Table 3 gives the results for the model with cross-unit cointegration, i.e. with a nonstationary common factor and stationary idiosyncratic components. The asymptotic tests have very large size distortions, and while the size distortions of the bootstrap tests are significantly less, they are still large. As expected it indeed seems that the bootstrap tests do not perform very well in this setting.
The problem partly arises, especially for the group-mean test, because for some units the loadings will be very close to zero, thereby making that unit effectively stationary and hence inflating the test statistic. In such a situation we may expect the median-based test to be more robust, and indeed it seems to perform somewhat better than the group-mean test although it still suffers from considerable size distortions.

Table 4 presents results for the model under the alternative without a common factor. The power of the bootstrap tests is satisfactory, and as expected, increases with both $T$ and $N$. The only setting in which we can directly compare the power of the asymptotic and the bootstrap tests is the setting of no dependence (Panel A), and here power results are very similar. Given that the bootstrap tests are somewhat undersized, this shows that the power of the bootstrap tests is good. In the other settings the power of the bootstrap test is somewhat less than the power of the asymptotic tests, which can be explained by the size distortions of the asymptotic tests. Note that the bootstrap tests perform similarly.

Table 5 gives results for power with a common factor. It can be seen that the power of the bootstrap tests still increases with $T$ and $N$, although the power is less than that in Table 4 and especially the increase in power with $N$ is less. This is not surprising as the common factor which is present in every unit ensures that 
Table 2

Size properties with common factors (setting II).

\begin{tabular}{|c|c|c|c|c|c|c|c|c|}
\hline$A_{1}, B_{1}$ & $\Sigma$ & $T$ & $N$ & $\tau_{l l c}$ & $\tau_{p}$ & $\tau_{i p s}$ & $\tau_{g m}$ & $\tau_{\text {med }}$ \\
\hline \multicolumn{9}{|c|}{ Panel A: No short-run dependence } \\
\hline$A_{1}=0$ & $r=1$ & 25 & 5 & 0.212 & 0.029 & 0.188 & 0.024 & 0.030 \\
\hline \multirow[t]{8}{*}{$B_{1}=0$} & & 25 & 25 & 0.288 & 0.014 & 0.337 & 0.015 & 0.023 \\
\hline & & 25 & 50 & 0.352 & 0.009 & 0.425 & 0.013 & 0.014 \\
\hline & & 50 & 5 & 0.165 & 0.036 & 0.104 & 0.030 & 0.037 \\
\hline & & 50 & 25 & 0.218 & 0.021 & 0.280 & 0.022 & 0.030 \\
\hline & & 50 & 50 & 0.263 & 0.018 & 0.362 & 0.020 & 0.023 \\
\hline & & 100 & 5 & 0.160 & 0.039 & 0.095 & 0.030 & 0.038 \\
\hline & & 100 & 25 & 0.213 & 0.030 & 0.258 & 0.025 & 0.035 \\
\hline & & 100 & 50 & 0.253 & 0.020 & 0.342 & 0.022 & 0.024 \\
\hline \multicolumn{9}{|c|}{ Panel B: Contemporaneous, but no dynamic dependence } \\
\hline$A_{1}=0$ & $r=0.1$ & 25 & 5 & 0.229 & 0.030 & 0.204 & 0.029 & 0.034 \\
\hline \multirow[t]{8}{*}{$B_{1}=0$} & & 25 & 25 & 0.314 & 0.025 & 0.389 & 0.021 & 0.027 \\
\hline & & 25 & 50 & 0.359 & 0.023 & 0.467 & 0.021 & 0.024 \\
\hline & & 50 & 5 & 0.193 & 0.036 & 0.122 & 0.031 & 0.036 \\
\hline & & 50 & 25 & 0.283 & 0.032 & 0.332 & 0.026 & 0.031 \\
\hline & & 50 & 50 & 0.296 & 0.027 & 0.393 & 0.025 & 0.028 \\
\hline & & 100 & 5 & 0.182 & 0.040 & 0.114 & 0.030 & 0.036 \\
\hline & & 100 & 25 & 0.277 & 0.034 & 0.316 & 0.030 & 0.038 \\
\hline & & 100 & 50 & 0.315 & 0.031 & 0.390 & 0.031 & 0.035 \\
\hline \multicolumn{9}{|c|}{ Panel C: Contemporaneous and dynamic dependence } \\
\hline$A_{1}=\Xi$ & $r=0.1$ & 25 & 5 & 0.269 & 0.022 & 0.243 & 0.023 & 0.022 \\
\hline \multirow[t]{8}{*}{$B_{1}=\Omega$} & & 25 & 25 & 0.351 & 0.012 & 0.381 & 0.013 & 0.016 \\
\hline & & 25 & 50 & 0.406 & 0.007 & 0.448 & 0.010 & 0.011 \\
\hline & & 50 & 5 & 0.253 & 0.025 & 0.177 & 0.050 & 0.024 \\
\hline & & 50 & 25 & 0.348 & 0.015 & 0.358 & 0.018 & 0.020 \\
\hline & & 50 & 50 & 0.378 & 0.013 & 0.411 & 0.016 & 0.021 \\
\hline & & 100 & 5 & 0.252 & 0.032 & 0.172 & 0.032 & 0.031 \\
\hline & & 100 & 25 & 0.373 & 0.023 & 0.362 & 0.025 & 0.028 \\
\hline & & 100 & 50 & 0.420 & 0.023 & 0.425 & 0.022 & 0.028 \\
\hline
\end{tabular}

See Table 1.

Table 3

Size properties with cross-unit cointegration (setting III).

\begin{tabular}{|c|c|c|c|c|c|c|c|c|}
\hline$A_{1}, B_{1}$ & $\Sigma$ & $T$ & $N$ & $\tau_{l l c}$ & $\tau_{p}$ & $\tau_{i p s}$ & $\tau_{g m}$ & $\tau_{\text {med }}$ \\
\hline \multicolumn{9}{|c|}{ Panel A: No short-run dependence } \\
\hline$A_{1}=0$ & $r=1$ & 25 & 5 & 0.410 & 0.129 & 0.332 & 0.103 & 0.102 \\
\hline \multirow[t]{8}{*}{$B_{1}=0$} & & 25 & 25 & 0.629 & 0.198 & 0.585 & 0.173 & 0.198 \\
\hline & & 25 & 50 & 0.698 & 0.224 & 0.643 & 0.173 & 0.216 \\
\hline & & 50 & 5 & 0.612 & 0.200 & 0.463 & 0.169 & 0.170 \\
\hline & & 50 & 25 & 0.782 & 0.267 & 0.620 & 0.259 & 0.251 \\
\hline & & 50 & 50 & 0.816 & 0.281 & 0.671 & 0.282 & 0.283 \\
\hline & & 100 & 5 & 0.674 & 0.240 & 0.550 & 0.333 & 0.257 \\
\hline & & 100 & 25 & 0.798 & 0.303 & 0.642 & 0.282 & 0.275 \\
\hline & & 100 & 50 & 0.845 & 0.336 & 0.688 & 0.399 & 0.336 \\
\hline \multicolumn{9}{|c|}{ Panel B: Contemporaneous, but no dynamic dependence } \\
\hline$A_{1}=0$ & $r=0.1$ & 25 & 5 & 0.461 & 0.096 & 0.356 & 0.086 & 0.084 \\
\hline \multirow[t]{8}{*}{$B_{1}=0$} & & 25 & 25 & 0.612 & 0.128 & 0.561 & 0.111 & 0.126 \\
\hline & & 25 & 50 & 0.667 & 0.166 & 0.598 & 0.148 & 0.177 \\
\hline & & 50 & 5 & 0.554 & 0.141 & 0.362 & 0.136 & 0.137 \\
\hline & & 50 & 25 & 0.742 & 0.179 & 0.552 & 0.183 & 0.184 \\
\hline & & 50 & 50 & 0.764 & 0.184 & 0.591 & 0.175 & 0.183 \\
\hline & & 100 & 5 & 0.651 & 0.171 & 0.431 & 0.187 & 0.149 \\
\hline & & 100 & 25 & 0.806 & 0.177 & 0.622 & 0.198 & 0.176 \\
\hline & & 100 & 50 & 0.819 & 0.211 & 0.633 & 0.253 & 0.210 \\
\hline \multicolumn{9}{|c|}{ Panel C: Contemporaneous and dynamic dependence } \\
\hline & $r=0.1$ & 25 & 5 & 0.427 & 0.047 & 0.357 & 0.049 & 0.041 \\
\hline \multirow{8}{*}{$B_{1}=\Omega$} & & 25 & 25 & 0.549 & 0.062 & 0.492 & 0.071 & 0.064 \\
\hline & & 25 & 50 & 0.597 & 0.067 & 0.522 & 0.065 & 0.068 \\
\hline & & 50 & 5 & 0.466 & 0.085 & 0.309 & 0.125 & 0.089 \\
\hline & & 50 & 25 & 0.670 & 0.098 & 0.516 & 0.122 & 0.092 \\
\hline & & 50 & 50 & 0.698 & 0.089 & 0.539 & 0.107 & 0.087 \\
\hline & & 100 & 5 & 0.464 & 0.100 & 0.305 & 0.136 & 0.115 \\
\hline & & 100 & 25 & 0.701 & 0.117 & 0.527 & 0.165 & 0.104 \\
\hline & & 100 & 50 & 0.738 & 0.109 & 0.575 & 0.148 & 0.101 \\
\hline
\end{tabular}

See Table 1. 
Table 4

Power properties without common factors (setting IV).

\begin{tabular}{|c|c|c|c|c|c|c|c|c|}
\hline$A_{1}, B_{1}$ & $\Sigma$ & $T$ & $N$ & $\tau_{l l c}$ & $\tau_{p}$ & $\tau_{\text {ips }}$ & $\tau_{g m}$ & $\tau_{\text {med }}$ \\
\hline \multicolumn{9}{|c|}{ Panel A: No short-run dependence } \\
\hline$A_{1}=0$ & $r=1$ & 25 & 5 & 0.607 & 0.507 & 0.651 & 0.354 & 0.337 \\
\hline \multirow{8}{*}{$B_{1}=0$} & & 25 & 25 & 0.829 & 0.866 & 0.980 & 0.894 & 0.892 \\
\hline & & 25 & 50 & 0.875 & 0.958 & 0.999 & 0.996 & 0.996 \\
\hline & & 50 & 5 & 0.754 & 0.757 & 0.829 & 0.810 & 0.773 \\
\hline & & 50 & 25 & 0.995 & 0.999 & 1.000 & 1.000 & 1.000 \\
\hline & & 50 & 50 & 1.000 & 1.000 & 1.000 & 1.000 & 1.000 \\
\hline & & 100 & 5 & 0.905 & 0.929 & 0.989 & 0.974 & 0.946 \\
\hline & & 100 & 25 & 1.000 & 1.000 & 1.000 & 1.000 & 1.000 \\
\hline & & 100 & 50 & 1.000 & 1.000 & 1.000 & 1.000 & 1.000 \\
\hline \multicolumn{9}{|c|}{ Panel B: Contemporaneous, but no dynamic dependence } \\
\hline & $r=0.1$ & 25 & 5 & 0.553 & 0.508 & 0.608 & 0.357 & 0.361 \\
\hline \multirow{8}{*}{$B_{1}=0$} & & 25 & 25 & 0.832 & 0.827 & 0.985 & 0.850 & 0.854 \\
\hline & & 25 & 50 & 0.878 & 0.919 & 1.000 & 0.981 & 0.980 \\
\hline & & 50 & 5 & 0.856 & 0.630 & 0.887 & 0.648 & 0.633 \\
\hline & & 50 & 25 & 0.998 & 0.989 & 1.000 & 1.000 & 0.999 \\
\hline & & 50 & 50 & 1.000 & 1.000 & 1.000 & 1.000 & 1.000 \\
\hline & & 100 & 5 & 0.928 & 0.943 & 0.996 & 0.985 & 0.950 \\
\hline & & 100 & 25 & 1.000 & 1.000 & 1.000 & 1.000 & 1.000 \\
\hline & & 100 & 50 & 1.000 & 1.000 & 1.000 & 1.000 & 1.000 \\
\hline \multicolumn{9}{|c|}{ Panel C: No contemporaneous, but dynamic dependence } \\
\hline & $r=1$ & 25 & 5 & 0.573 & 0.491 & 0.625 & 0.498 & 0.391 \\
\hline \multirow{8}{*}{$B_{1}=\Omega$} & & 25 & 25 & 0.798 & 0.618 & 0.972 & 0.862 & 0.768 \\
\hline & & 25 & 50 & 0.866 & 0.711 & 0.998 & 0.984 & 0.934 \\
\hline & & 50 & 5 & 0.816 & 0.700 & 0.865 & 0.779 & 0.675 \\
\hline & & 50 & 25 & 0.991 & 0.955 & 1.000 & 1.000 & 0.997 \\
\hline & & 50 & 50 & 1.000 & 0.983 & 1.000 & 1.000 & 1.000 \\
\hline & & 100 & 5 & 0.896 & 0.891 & 0.975 & 0.950 & 0.955 \\
\hline & & 100 & 25 & 1.000 & 0.996 & 1.000 & 1.000 & 1.000 \\
\hline & & 100 & 50 & 1.000 & 0.999 & 1.000 & 1.000 & 1.000 \\
\hline \multicolumn{9}{|c|}{ Panel D: Contemporaneous and dynamic dependence } \\
\hline \multirow{9}{*}{$\begin{array}{l}A_{1}=\Xi \\
B_{1}=\Omega\end{array}$} & $r=0.1$ & 25 & 5 & 0.575 & 0.354 & 0.609 & 0.413 & 0.361 \\
\hline & & 25 & 25 & 0.767 & 0.754 & 0.949 & 0.860 & 0.820 \\
\hline & & 25 & 50 & 0.841 & 0.729 & 0.997 & 0.981 & 0.941 \\
\hline & & 50 & 5 & 0.755 & 0.693 & 0.814 & 0.729 & 0.607 \\
\hline & & 50 & 25 & 0.989 & 0.934 & 1.000 & 0.999 & 0.996 \\
\hline & & 50 & 50 & 0.999 & 0.989 & 1.000 & 1.000 & 1.000 \\
\hline & & 100 & 5 & 0.985 & 0.762 & 0.994 & 0.884 & 0.830 \\
\hline & & 100 & 25 & 0.999 & 0.995 & 1.000 & 1.000 & 1.000 \\
\hline & & 100 & 50 & 1.000 & 1.000 & 1.000 & 1.000 & 1.000 \\
\hline
\end{tabular}

See Table 1.

the information on the order of integration is not increased by much by the addition of units in the panel. The fact that the power of the asymptotic tests is higher than the power of the bootstrap tests can be explained by the large size distortions of the asymptotic tests in this case. The bootstrap tests all have similar power properties, although the median-based test seems to be somewhat less powerful than the group-mean test.

To conclude, we can see that the bootstrap tests have reasonable finite sample properties, with the exception of the oversize in the cross-unit cointegration setting and, to a lesser extent, the undersize in the setting without common factors for increasing $N .^{15}$

\footnotetext{
$\overline{15}$ In order to compare the finite sample performance of our tests to that of factor-based tests, we consider the simulation study of Gengenbach et al. (2010), which uses a DGP that is fairly similar to ours (with the exception of the shortrun dynamics). We see that many of the tests considered there also suffer from size distortions, even in the setting with both factors and idiosyncratic components $I(1)$. In the cross-unit cointegration setting most tests, with the exception of the tests by Bai and $\mathrm{Ng}$ (2004), suffer from severe size distortions. It seems that no test considered in that simulation study generally outperforms our bootstrap tests, both in terms of size and power. We can also compare the bootstrap tests to the subsampling tests of Choi and Chue (2007), which in a way are the only tests that are directly comparable to ours in terms of underlying assumptions. Their simulation study shows that the subsampling tests have good size properties, and they clearly
}

\subsection{Block length selection}

The Monte Carlo experiment in the previous section was done with fixed block lengths. It is well known from the literature on block bootstrap that the block length selected can have a large effect on the performance of any kind of application of the block bootstrap. That is of course valid here as well. Added to the usual issues relating to the structure of the temporal dependence, block length selection is also important in our setting in the case of cross-unit cointegration, where one can expect that large blocks are needed based on the discussion in Section 4.2.2. Our discussion here mirrors the discussion in Paparoditis and Politis (2003, Section 6.1), who discuss the selection of block lengths for univariate unit root tests.

Quite some research has been done on optimal block length selection in the framework of stationary time series. As noted in Paparoditis and Politis (2003) in order to talk about optimality one needs to set a criterion that is to be optimized. This criterion will depend on the type of application of the bootstrap (variance estimation, confidence intervals, hypothesis tests, etc.). Using

outperform our bootstrap tests in the case of cross-unit cointegration. However, the DGPs employed by them are not completely comparable to ours in terms of shortrun dynamics and factor loadings. 
Table 5

Power properties with common factors (setting V).

\begin{tabular}{|c|c|c|c|c|c|c|c|c|}
\hline$A_{1}, B_{1}$ & $\Sigma$ & $T$ & $N$ & $\tau_{l l c}$ & $\tau_{p}$ & $\tau_{i p s}$ & $\tau_{g m}$ & $\tau_{\text {med }}$ \\
\hline \multicolumn{9}{|c|}{ Panel A: No short-run dependence } \\
\hline$A_{1}=0$ & $r=1$ & 25 & 5 & 0.597 & 0.213 & 0.512 & 0.173 & 0.177 \\
\hline \multirow{8}{*}{$B_{1}=0$} & & 25 & 25 & 0.784 & 0.345 & 0.795 & 0.306 & 0.344 \\
\hline & & 25 & 50 & 0.840 & 0.377 & 0.858 & 0.326 & 0.365 \\
\hline & & 50 & 5 & 0.883 & 0.505 & 0.783 & 0.504 & 0.474 \\
\hline & & 50 & 25 & 0.989 & 0.677 & 0.957 & 0.672 & 0.658 \\
\hline & & 50 & 50 & 0.997 & 0.723 & 0.974 & 0.750 & 0.722 \\
\hline & & 100 & 5 & 0.978 & 0.822 & 0.970 & 0.830 & 0.802 \\
\hline & & 100 & 25 & 1.000 & 0.944 & 1.000 & 0.964 & 0.933 \\
\hline & & 100 & 50 & 1.000 & 0.956 & 0.999 & 0.981 & 0.961 \\
\hline \multicolumn{9}{|c|}{ Panel B: Contemporaneous, but no dynamic dependence } \\
\hline$A_{1}=0$ & $r=0.1$ & 25 & 5 & 0.590 & 0.189 & 0.506 & 0.140 & 0.148 \\
\hline \multirow[t]{8}{*}{$B_{1}=0$} & & 25 & 25 & 0.767 & 0.265 & 0.772 & 0.236 & 0.263 \\
\hline & & 25 & 50 & 0.822 & 0.271 & 0.812 & 0.234 & 0.266 \\
\hline & & 50 & 5 & 0.828 & 0.415 & 0.682 & 0.423 & 0.399 \\
\hline & & 50 & 25 & 0.981 & 0.503 & 0.935 & 0.487 & 0.497 \\
\hline & & 50 & 50 & 0.994 & 0.497 & 0.958 & 0.509 & 0.508 \\
\hline & & 100 & 5 & 0.963 & 0.695 & 0.928 & 0.792 & 0.731 \\
\hline & & 100 & 25 & 1.000 & 0.840 & 0.996 & 0.883 & 0.834 \\
\hline & & 100 & 50 & 1.000 & 0.853 & 0.998 & 0.912 & 0.850 \\
\hline \multicolumn{9}{|c|}{ Panel C: Contemporaneous and dynamic dependence } \\
\hline$A_{1}=\Xi$ & $r=0.1$ & 25 & 5 & 0.534 & 0.078 & 0.480 & 0.087 & 0.074 \\
\hline \multirow{8}{*}{$B_{1}=\Omega$} & & 25 & 25 & 0.707 & 0.096 & 0.674 & 0.085 & 0.100 \\
\hline & & 25 & 50 & 0.753 & 0.124 & 0.720 & 0.126 & 0.124 \\
\hline & & 50 & 5 & 0.801 & 0.243 & 0.669 & 0.244 & 0.190 \\
\hline & & 50 & 25 & 0.953 & 0.270 & 0.876 & 0.329 & 0.253 \\
\hline & & 50 & 50 & 0.972 & 0.272 & 0.903 & 0.328 & 0.276 \\
\hline & & 100 & 5 & 0.967 & 0.482 & 0.904 & 0.619 & 0.454 \\
\hline & & 100 & 25 & 0.998 & 0.527 & 0.987 & 0.712 & 0.494 \\
\hline & & 100 & 50 & 0.999 & 0.596 & 0.989 & 0.756 & 0.564 \\
\hline
\end{tabular}

See Table 1.

higher order asymptotics, it has been found for stationary series that an optimal block length $b_{\text {opt }}$ is of the form

$b_{\mathrm{opt}}=C T^{1 / \kappa}$,

where $\kappa$ is a known integer depending on the type of application and $C$ is usually unknown and depends on the data. Härdle et al. (2003) and Lahiri (2003) give an overview on optimal block lengths in stationary time series.

Several methods have been proposed in the setting where one can describe $b_{\text {opt }}$ as in (27). Some are based on the estimation of $C$ by exploiting the dependence of $C$ on certain quantities that can be estimated. Bühlmann and Künsch (1999) and Politis and White (2004) are examples of such methods that are applicable for variance estimation. Lahiri et al. (2007) propose a plug-in method, based on the jackknife-after-bootstrap, that is also applicable for confidence intervals and hypothesis tests.

A different method is the subsampling approach by Hall et al. (1995). The attractive feature of this method is that it avoids the estimation of $C$. This feature, as well as the ease of its implementation, has made this method a popular choice among practitioners. It does however require knowledge of $\kappa$ to implement it.

The problem with nonstationary time series is that $\kappa$ is unknown here, as the required asymptotic expansions have not been developed yet. This makes it very difficult to implement any of the methods discussed above using a well founded choice of $\kappa$. Paparoditis and Politis (2003) discuss this issue and suggest some heuristic ideas to determine $\kappa$.

Alternative strategies to the methods discussed above are provided by the minimum volatility method and calibration method proposed by Politis et al. (1999). These methods do not require knowledge of $\kappa$. The minimum volatility method involves calculating critical values using a range of block lengths and selecting the optimal one in the region where the critical values have the lowest volatility.
We will focus here on the calibration method. ${ }^{16}$ In particular, we will consider the Warp-Speed calibration method, which is a modification of the original calibration method by Giacomini et al. (2007) for the purpose of constructing confidence intervals. We present the procedure for hypothesis tests below for completeness (also see Remark 9).

\section{Block length selection by Warp-Speed calibration}

1. Choose a starting value $b_{0}$ for the block length. Using this value, generate $K$ bootstrap samples: $\left(\left\{y_{t}^{1}\right\}, \ldots,\left\{y_{t}^{K}\right\}\right)$. Calculate the statistic of interest for each bootstrap sample, say $\hat{\theta}^{k}\left(b_{0}\right)$ for $k=1, \ldots, K$. Using the empirical distribution of the statistics, calculate the bootstrap critical value $c\left(b_{0}\right)$.

2. Let $\left(b_{1}, \ldots, b_{M}\right)$ be the candidate block lengths. For each $i=$ $1, \ldots, M$ and $k=1, \ldots, K$, construct one bootstrap resample from the bootstrap sample $\left\{y_{t}^{k}\right\}$ using block length $b_{i}$, call this $\left\{y_{t}^{k}(i)\right\}$. Using each resample calculate the statistic of interest, say $\hat{\theta}^{* k}\left(b_{i}\right)$.

3. Using the distribution of $\hat{\theta}^{* k}\left(b_{i}\right)$ for $k=1, \ldots, K$, calculate the bootstrap resample critical value $c^{*}\left(b_{i}\right)$ for all $i=1, \ldots, M$.

4. Select the optimal block length $b_{\mathrm{opt}}$ such that

$$
b_{\mathrm{opt}}=\underset{b_{i}, i=1 \ldots, M}{\arg \min }\left|c^{*}\left(b_{i}\right)-c\left(b_{0}\right)\right| .
$$

To reduce the dependence on $b_{0}$ one can apply this algorithm iteratively, by using $b_{\text {opt }}$ as the starting block length in the next iteration and continuing until convergence.

\footnotetext{
16 We also considered the minimum volatility method, the subsampling method by Hall et al. (1995) and the plug-in method by Lahiri et al. (2007), the latter two with the value for $\kappa$ based on the results for stationary time series, but all these methods were inferior to the calibration method; see Remark 10.
} 
Table 6

Size properties with block length selection.

\begin{tabular}{|c|c|c|c|c|c|c|c|c|c|c|c|}
\hline \multirow[t]{2}{*}{$A_{1}, B_{1}$} & \multirow[t]{2}{*}{$\Sigma$} & \multirow[t]{2}{*}{$T$} & \multirow[t]{2}{*}{$N$} & \multicolumn{4}{|l|}{$\tau_{p}$} & \multicolumn{4}{|l|}{$\tau_{g m}$} \\
\hline & & & & $\mathrm{RF}$ & AvB & OpB & OpRF & $\mathrm{RF}$ & AvB & OpB & OpRF \\
\hline \multicolumn{12}{|c|}{ Panel A: No common factors (setting I) } \\
\hline$A_{1}=0$ & $r=1$ & 25 & 5 & 0.022 & 3.992 & 1 & 0.038 & 0.018 & 4.172 & 1 & 0.038 \\
\hline \multirow{7}{*}{$B_{1}=0$} & & 25 & 25 & 0.002 & 2.778 & 1 & 0.012 & 0.012 & 2.762 & 2 & 0.038 \\
\hline & & 50 & 5 & 0.028 & 6.472 & 5 & 0.050 & 0.020 & 6.468 & 2 & 0.050 \\
\hline & & 50 & 25 & 0.012 & 3.610 & 1 & 0.020 & 0.014 & 3.760 & 1 & 0.038 \\
\hline & $r=0.1$ & 25 & 5 & 0.044 & 4.414 & 4 & 0.050 & 0.014 & 4.378 & 2 & 0.050 \\
\hline & & 25 & 25 & 0.010 & 2.944 & 1 & 0.014 & 0.012 & 2.912 & 1 & 0.062 \\
\hline & & 50 & 5 & 0.034 & 6.990 & 1 & 0.052 & 0.034 & 6.482 & 5 & 0.054 \\
\hline & & 50 & 25 & 0.016 & 3.658 & 1 & 0.024 & 0.014 & 3.672 & 3 & 0.038 \\
\hline$A_{1}=\Xi$ & $r=1$ & 25 & 5 & 0.032 & 4.056 & 2 & 0.038 & 0.012 & 4.504 & 2 & 0.044 \\
\hline \multirow[t]{7}{*}{$B_{1}=\Omega$} & & 25 & 25 & 0.002 & 2.304 & 1 & 0.008 & 0.008 & 2.332 & 2 & 0.032 \\
\hline & & 50 & 5 & 0.042 & 7.968 & 6 & 0.052 & 0.024 & 9.906 & 13 & 0.050 \\
\hline & & 50 & 25 & 0.006 & 2.918 & 3 & 0.010 & 0.046 & 4.144 & 5 & 0.052 \\
\hline & $r=0.1$ & 25 & 5 & 0.024 & 4.502 & 4 & 0.048 & 0.042 & 5.066 & 10 & 0.042 \\
\hline & & 25 & 25 & 0.008 & 2.578 & 1 & 0.024 & 0.028 & 2.968 & 3 & 0.032 \\
\hline & & 50 & 5 & 0.042 & 7.212 & 6 & 0.054 & 0.036 & 7.092 & 4 & 0.050 \\
\hline & & 50 & 25 & 0.008 & 3.314 & 5 & 0.012 & 0.010 & 3.434 & 2 & 0.020 \\
\hline \multicolumn{12}{|c|}{ Panel B: Common factors (setting II) } \\
\hline$A_{1}=0$ & $r=1$ & 25 & 5 & 0.022 & 4.878 & 2 & 0.046 & 0.020 & 4.950 & 2 & 0.052 \\
\hline \multirow[t]{7}{*}{$B_{1}=0$} & & 25 & 25 & 0.018 & 3.510 & 2 & 0.042 & 0.008 & 3.286 & 1 & 0.060 \\
\hline & & 50 & 5 & 0.018 & 8.850 & 12 & 0.050 & 0.034 & 7.086 & 5 & 0.050 \\
\hline & & 50 & 25 & 0.018 & 5.566 & 2 & 0.046 & 0.024 & 5.064 & 1 & 0.050 \\
\hline & $r=0.1$ & 25 & 5 & 0.028 & 5.494 & 6 & 0.046 & 0.020 & 4.938 & 1 & 0.048 \\
\hline & & 25 & 25 & 0.016 & 3.978 & 1 & 0.038 & 0.004 & 4.154 & 2 & 0.048 \\
\hline & & 50 & 5 & 0.020 & 10.292 & 5 & 0.048 & 0.032 & 8.892 & 2 & 0.056 \\
\hline & & 50 & 25 & 0.044 & 7.154 & 4 & 0.050 & 0.038 & 6.098 & 4 & 0.048 \\
\hline$A_{1}=\Xi$ & $r=0.1$ & 25 & 5 & 0.006 & 4.946 & 3 & 0.020 & 0.004 & 5.128 & 5 & 0.014 \\
\hline \multirow{3}{*}{$B_{1}=\Omega$} & & 25 & 25 & 0.000 & 4.572 & 3 & 0.038 & 0.006 & 4.558 & 6 & 0.026 \\
\hline & & 50 & 5 & 0.010 & 8.606 & 2 & 0.036 & 0.012 & 8.802 & 7 & 0.028 \\
\hline & & 50 & 25 & 0.014 & 6.270 & 3 & 0.018 & 0.016 & 5.532 & 6 & 0.024 \\
\hline \multicolumn{12}{|c|}{ Panel C: Cross-unit cointegration (setting III) } \\
\hline & $r=1$ & 25 & 5 & 0.098 & 5.442 & 16 & 0.054 & 0.128 & 5.038 & 16 & 0.076 \\
\hline \multirow{7}{*}{$B_{1}=0$} & & 25 & 25 & 0.192 & 4.208 & 17 & 0.078 & 0.144 & 3.976 & 19 & 0.070 \\
\hline & & 50 & 5 & 0.126 & 9.266 & 30 & 0.050 & 0.108 & 8.056 & 27 & 0.052 \\
\hline & & 50 & 25 & 0.230 & 5.984 & 37 & 0.090 & 0.266 & 5.140 & 33 & 0.114 \\
\hline & $r=0.1$ & 25 & 5 & 0.024 & 6.626 & 5 & 0.046 & 0.018 & 6.394 & 1 & 0.052 \\
\hline & & 25 & 25 & 0.152 & 4.296 & 15 & 0.056 & 0.102 & 4.122 & 15 & 0.052 \\
\hline & & 50 & 5 & 0.044 & 11.222 & 10 & 0.048 & 0.038 & 9.448 & 26 & 0.052 \\
\hline & & 50 & 25 & 0.180 & 7.512 & 35 & 0.062 & 0.132 & 6.572 & 24 & 0.076 \\
\hline \multirow{4}{*}{$\begin{array}{l}A_{1}=\Xi \\
B_{1}=\Omega\end{array}$} & $r=0.1$ & 25 & 5 & 0.046 & 5.140 & 5 & 0.050 & 0.044 & 4.756 & 8 & 0.052 \\
\hline & & 25 & 25 & 0.082 & 4.044 & 10 & 0.046 & 0.114 & 3.734 & 14 & 0.050 \\
\hline & & 50 & 5 & 0.032 & 9.536 & 6 & 0.048 & 0.018 & 8.686 & 3 & 0.040 \\
\hline & & 50 & 25 & 0.070 & 7.192 & 1 & 0.052 & 0.086 & 6.266 & 20 & 0.052 \\
\hline
\end{tabular}

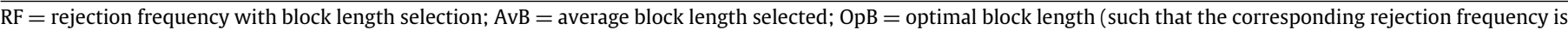
as close as possible to 0.05$) ; \mathrm{OpRF}=$ rejection frequency corresponding to the optimal block length.

To analyze the performance of the method, we performed a small Monte Carlo experiment using the same DGP as in Section 5.1 applying the tests $\tau_{p}$ and $\tau_{g m}$. Based on 500 simulations, we let the block length be selected by the Warp-Speed calibration method, and using the same seed, we run the tests for a wide range of fixed block lengths (up to 0.75 times the sample size) to determine the optimal block length. As starting block lengths we take the fixed block lengths from the previous section, while we take $K=199$. Due to computational costs we do not iterate the algorithm.

Results for size are given in Table 6. Optimal block lengths are determined as those block lengths which give an empirical rejection frequency the closest to the nominal level (5\%). It can be seen that while the optimal rejection frequencies are not obtained using the block length selection method, the rejection frequencies for setting I and II are reasonably close. However, while the selected block lengths do increase for setting III, they do not increase sufficiently compared to the optimal block lengths and size distortions persist.

Results for power are presented in Table 7. Optimal block lengths here are selected as the block lengths that give the highest power possible. One should regard this with caution, as optimal block lengths under the alternative hypothesis are difficult to define, as higher power could come at the expense of good size properties under the null. It is therefore not clear whether high power is the criterion that should be optimized. ${ }^{17}$ What is clear though, is that choosing an unnecessarily large block length will decrease power. ${ }^{18}$ The results show that the calibration method performs reasonably satisfactorily.

To conclude, using the calibration method improves on using a fixed block length, but it is not optimal. A lot of work still needs to be done on this topic, especially from a theoretical perspective.

Remark 9. The equivalent for hypothesis testing of the method proposed by Giacomini et al. (2007) for confidence intervals would

\footnotetext{
17 Note that even when using size-adjusted power this problem would still be present.

18 As pointed out by one of the referees, this may be due to the fact that for a large block length the possible bootstrap samples, and correspondingly the possible bootstrap statistics will be more alike to each other and to the original sample (statistics). This will lead to a poorer approximation of the bootstrap distribution, and eventually to a loss of power.
} 
Table 7

Power properties with block length selection.

\begin{tabular}{|c|c|c|c|c|c|c|c|c|c|c|c|}
\hline \multirow[t]{2}{*}{$A_{1}, B_{1}$} & \multirow[t]{2}{*}{$\Sigma$} & \multirow[t]{2}{*}{$T$} & \multirow[t]{2}{*}{$N$} & \multicolumn{4}{|l|}{$\tau_{p}$} & \multicolumn{4}{|l|}{$\underline{\tau_{g m}}$} \\
\hline & & & & $\mathrm{RF}$ & AvB & OpB & OpRF & $\mathrm{RF}$ & AvB & OpB & OpRF \\
\hline \multicolumn{12}{|c|}{ Panel A: No common factors (setting IV) } \\
\hline$A_{1}=0$ & $r=1$ & 25 & 5 & 0.218 & 4.088 & 2 & 0.436 & 0.186 & 4.060 & 2 & 0.244 \\
\hline \multirow{7}{*}{$B_{1}=0$} & & 25 & 25 & 0.916 & 2.928 & 1 & 0.964 & 0.986 & 2.596 & 1 & 0.990 \\
\hline & & 50 & 5 & 0.740 & 5.974 & 5 & 0.856 & 0.884 & 5.438 & 4 & 0.948 \\
\hline & & 50 & 25 & 1.000 & 3.732 & 1 & 1.000 & 1.000 & 3.168 & 1 & 1.000 \\
\hline & $r=0.1$ & 25 & 5 & 0.686 & 4.530 & 4 & 0.816 & 0.340 & 4.194 & 1 & 0.636 \\
\hline & & 25 & 25 & 0.984 & 2.868 & 1 & 0.992 & 0.966 & 2.606 & 2 & 0.976 \\
\hline & & 50 & 5 & 0.652 & 6.592 & 5 & 0.792 & 0.748 & 5.730 & 3 & 0.850 \\
\hline & & 50 & 25 & 1.000 & 3.766 & 1 & 1.000 & 1.000 & 3.118 & 1 & 1.000 \\
\hline$A_{1}=\Xi$ & $r=1$ & 25 & 5 & 0.102 & 3.788 & 6 & 0.132 & 0.192 & 3.908 & 4 & 0.270 \\
\hline \multirow[t]{7}{*}{$B_{1}=\Omega$} & & 25 & 25 & 0.476 & 2.458 & 4 & 0.428 & 0.756 & 2.390 & 3 & 0.860 \\
\hline & & 50 & 5 & 0.582 & 7.586 & 4 & 0.682 & 0.570 & 7.412 & 1 & 0.798 \\
\hline & & 50 & 25 & 0.988 & 3.192 & 2 & 0.992 & 1.000 & 2.850 & 1 & 1.000 \\
\hline & $r=0.1$ & 25 & 5 & 0.234 & 4.076 & 4 & 0.330 & 0.250 & 3.966 & 3 & 0.400 \\
\hline & & 25 & 25 & 0.642 & 2.414 & 2 & 0.704 & 0.784 & 2.390 & 3 & 0.880 \\
\hline & & 50 & 5 & 0.264 & 6.976 & 3 & 0.410 & 0.198 & 6.702 & 4 & 0.354 \\
\hline & & 50 & 25 & 0.998 & 3.362 & 2 & 1.000 & 1.000 & 2.994 & 1 & 1.000 \\
\hline \multicolumn{12}{|c|}{ Panel B: Common factors (setting V) } \\
\hline \multirow{8}{*}{$\begin{array}{l}A_{1}=0 \\
B_{1}=0\end{array}$} & $r=1$ & 25 & 5 & 0.118 & 4.712 & 1 & 0.300 & 0.138 & 4.386 & 1 & 0.254 \\
\hline & & 25 & 25 & 0.328 & 3.802 & 1 & 0.550 & 0.242 & 3.608 & 2 & 0.402 \\
\hline & & 50 & 5 & 0.340 & 8.188 & 3 & 0.486 & 0.372 & 6.868 & 3 & 0.438 \\
\hline & & 50 & 25 & 0.538 & 6.338 & 1 & 0.682 & 0.484 & 5.638 & 2 & 0.684 \\
\hline & $r=0.1$ & 25 & 5 & 0.226 & 4.652 & 2 & 0.406 & 0.160 & 4.484 & 1 & 0.268 \\
\hline & & 25 & 25 & 0.182 & 4.342 & 1 & 0.250 & 0.100 & 4.276 & 2 & 0.240 \\
\hline & & 50 & 5 & 0.182 & 9.964 & 3 & 0.292 & 0.214 & 8.812 & 1 & 0.364 \\
\hline & & 50 & 25 & 0.414 & 7.096 & 1 & 0.508 & 0.580 & 5.858 & 1 & 0.752 \\
\hline \multirow{4}{*}{$\begin{array}{l}A_{1}=\Xi \\
B_{1}=\Omega\end{array}$} & $r=0.1$ & 25 & 5 & 0.064 & 5.120 & 8 & 0.120 & 0.058 & 4.574 & 2 & 0.136 \\
\hline & & 25 & 25 & 0.058 & 4.638 & 3 & 0.092 & 0.042 & 4.108 & 5 & 0.096 \\
\hline & & 50 & 5 & 0.110 & 8.774 & 2 & 0.184 & 0.098 & 8.166 & 3 & 0.158 \\
\hline & & 50 & 25 & 0.200 & 6.424 & 7 & 0.286 & 0.258 & 5.508 & 8 & 0.288 \\
\hline
\end{tabular}

See Table 6 .

involve a criterion that minimizes size distortions instead of critical values as we do. To be more specific, after Step 3 of the algorithm calculate the rejection frequency given block length $b_{i}$ using the bootstrap critical value $c^{*}\left(b_{i}\right)$ and the test statistics $\hat{\theta}^{k}\left(b_{0}\right)$. Then the optimal block length can be selected as that block length that makes the rejection frequency the closest to the nominal level.

While this criterion may seem to differ from our criterion, it is actually the same. We suggest minimizing the distance to $c\left(b_{0}\right)$, which is actually that value which, if used as a critical value, will exactly give a rejection frequency equal to the nominal level. Therefore, both criteria minimize the same thing. ${ }^{19}$ Moreover, our criterion may perform better if only a small number of replications is considered. For a small number of bootstrap replications, there are also only a limited number of values the rejection frequencies can take, with the consequence that the rejection frequency for several candidate block lengths will be the same and a number of block lengths may turn out to be optimal. Our criterion does not suffer from that problem, as the critical values are not restricted in this way.

Remark 10. As mentioned before, we compared the calibration method to the subsampling approach of Hall et al. (1995), the plug-in method of Lahiri et al. (2007) and the minimum volatility method. The subsampling method tends to select block lengths in a somewhat unpredictable way, although the obtained rejection frequencies are reasonably close (but somewhat inferior) to those obtained with the calibration method. The plug-in method generally favors too small block lengths, regardless of

19 Loosely speaking, we can describe this situation as a $p$-value approach versus a critical value approach, where both obviously lead to the same conclusion. the underlying DGP. The minimum volatility method selects block lengths almost uniformly over the range of allowed lengths, thereby selecting too large block lengths in general. The results are available on request.

\section{Conclusion}

We have established the asymptotic validity of two block bootstrap panel unit root tests for a model that includes various kinds of cross-sectional and temporal dependence. This includes a common factor structure and possibly cross-unit cointegration. The tests are simple pooled and group-mean tests based on the popular LLC and IPS tests. The finite sample properties of our test statistics have also been investigated and shown to be satisfactory in general. There also seems to be little difference between the bootstrap tests considered.

While for most specific settings (in particular cross-unit cointegration) some tests can be found that perform better for that particular setting, it is a lot more difficult to find a test that is valid for all the settings for which our bootstrap tests are valid. Moreover, there are currently very few tests that are valid in the empirically relevant case of dynamic cross-sectional dependence, while our tests are valid even in that setting. Our tests are very easy to implement as no specification and estimation of the dependence structure is necessary, and will therefore be very useful for practice when the true form of the cross-sectional (and temporal) dependence is not known and robustness to the unknown cross-sectional dependence matters. In fact, quite a lot of practitioners already use the block bootstrap to account for crosssectional dependence for the reasons listed above. Hence, this work provides the necessary theoretical justification.

On the basis of the theoretical and simulation results in this paper, we conclude that it is legitimate to use the proposed tests 
in practice when testing for unit roots in the observed data of a panel of fixed $N$ entities, in the presence of various forms of crosssectional dependence. The block bootstrap algorithm described in Section 3 can be straightforwardly implemented whereby block lengths can be selected using the Warp-Speed calibration method.

This study still leaves several ends open. First, while we briefly considered the subject of block length selection, much still needs to be done as at the moment there does not exist a fully satisfactory method to select block lengths. Second, while our derivations do not depend on small $N$ in any way, it will be interesting to see what happens if $N \rightarrow \infty$. As explained, such a theoretical analysis is difficult in our setting but it is certainly worth further research. Third, the specification of deterministic components remains an open issue. While a "naive" implementation of deterministic components is quite straightforward, and can even be seen to be valid without too much difficulty, experience has shown that including "naive" deterministic terms in panels is hardly ever a good solution. Thus, further investigation of this issue is also merited. Finally, the block bootstrap may also be used for the analysis of panel cointegration, as done by Fachin (2007) and Di Iorio and Fachin (2008). To develop the appropriate theoretical foundations in that setting would be a logical generalization of this work.

\section{Acknowledgements}

Previous versions of this paper were presented at the conference on Factor Structures for Panel and Multivariate Time Series Data in Maastricht, the Econometric Society European Meeting 2009 in Barcelona and at seminars at the Econometric Institute, Lund University, CORE, the University of Cambridge and Louisiana State University. We thank the conference and seminar participants, Stefano Fachin, Anders Swensen, the associate editors Jörg Breitung and Joakim Westerlund and two anonymous referees for helpful comments and suggestions. We thank NWO and the Royal Netherlands Academy of Arts and Sciences for financial support. The usual disclaimer applies.

\section{Appendix}

Proof of Lemma 1. Note that by Assumption $1 W_{T}(r)=T^{-1 / 2}$ $\sum_{t=1}^{\lfloor T r\rfloor} \varepsilon_{t} \stackrel{d}{\rightarrow} \Sigma^{1 / 2} W(r)$. Then it follows from standard asymptotic theory for linear processes (see for example Phillips and Solo, 1992) that, uniformly in $r$,

$T^{-1 / 2} \sum_{t=1}^{\lfloor T r\rfloor} x_{t}=\Psi(1) W_{T}(r)+o_{p}(1)$,

and consequently $T^{-1 / 2} \sum_{t=1}^{\lfloor T r\rfloor} x_{t} \stackrel{d}{\rightarrow} \Psi(1) \Sigma^{1 / 2} W(r)$. The result then follows by the continuous mapping theorem.

To prove Theorem 1 we need some moments that appear in the asymptotic distributions.

Lemma A.1. Let $y_{t}$ be generated under $H_{0}$ setting (A). Let Assumption 1 hold. Then

(i) $\begin{aligned} & \Omega=\lim _{T \rightarrow \infty} T^{-1} \mathrm{E}\left(\sum_{t=1}^{T} u_{t}\right)\left(\sum_{t=1}^{T} u_{t}\right)^{\prime} \\ & =\Gamma^{\prime} \Psi(1) \Sigma \Psi(1)^{\prime} \Gamma,\end{aligned}$

(ii) $\Omega_{0}=\lim _{T \rightarrow \infty} T^{-1} \sum_{t=1}^{T} \mathrm{E} u_{t} u_{t}^{\prime}=\sum_{j=0}^{\infty} \Gamma^{\prime} \Psi_{j} \Sigma \Psi_{j}^{\prime} \Gamma$.
Proof of Lemma A.1. For part (i), note that

$$
\begin{aligned}
\Omega & =\lim _{T \rightarrow \infty} T^{-1} \sum_{s=1}^{T} \sum_{t=1}^{T} \mathrm{E} u_{s} u_{t}^{\prime} \\
& =\lim _{T \rightarrow \infty} T^{-1} \sum_{s=1}^{T} \sum_{t=1}^{T} \sum_{i=0}^{\infty} \sum_{j=0}^{\infty} \Gamma^{\prime} \Psi_{i} \mathrm{E} \varepsilon_{s-i} \varepsilon_{t-j}^{\prime} \Psi_{j}^{\prime} \Gamma \\
& =\sum_{i=0}^{\infty} \sum_{j=0}^{\infty} \Gamma^{\prime} \Psi_{i} \Sigma \Psi_{j}^{\prime} \Gamma=\Gamma^{\prime} \Psi(1) \Sigma \Psi(1)^{\prime} \Gamma .
\end{aligned}
$$

For part (ii) we have

$$
\begin{aligned}
\Omega_{0} & =\lim _{T \rightarrow \infty} T^{-1} \sum_{t=1}^{T} E\left(\sum_{j=0}^{\infty} \Gamma^{\prime} \Psi_{j} \varepsilon_{t-j}\right)\left(\sum_{j=0}^{\infty} \Gamma^{\prime} \Psi_{j} \varepsilon_{t-j}\right)^{\prime} \\
& =\lim _{T \rightarrow \infty} T^{-1} \sum_{t=1}^{T} \sum_{i=0}^{\infty} \sum_{j=0}^{\infty} \Gamma^{\prime} \Psi_{i} \mathrm{E} \varepsilon_{t-i} \varepsilon_{t-j}^{\prime} \Psi_{j}^{\prime} \Gamma \\
& =\sum_{i=0}^{\infty} \Gamma^{\prime} \Psi_{i} \Sigma \Psi_{i}^{\prime} \Gamma .
\end{aligned}
$$

This completes the proof.

Lemma A.2. Let $y_{t}$ be generated under $H_{0}$ setting (A). Let Assumption 1 hold. Then, as $T \rightarrow \infty$, we have for $i=1, \ldots, N$,

(i) $T^{-1} \sum_{t=1}^{T} y_{i, t-1} \Delta y_{i, t} \stackrel{d}{\rightarrow} \int_{0}^{1} B_{i}(r) \mathrm{d} B_{i}(r)+\frac{1}{2}\left(\omega_{i}-\omega_{0, i}\right)$,

(ii) $T^{-2} \sum_{t=1}^{T} y_{i, t-1}^{2} \stackrel{d}{\rightarrow} \int_{0}^{1} B_{i}(r)^{2} \mathrm{~d} r$,

where convergence also holds jointly.

Proof of Lemma A.2. The proof follows directly from Lemmas 1, A.1 and the continuous mapping theorem. Joint convergence can be established using the Cramér-Wold device (cf. Davidson, 2002, Theorem 25.5).

Proof of Theorem 1. The proof follows immediately from Lemma A.2.

In order to derive the bootstrap invariance principle we need three preliminary lemmas that build on each other. We exploit the linearity of the processes in our derivation. As for the original series, we first derive the properties for the bootstrap equivalent of $\varepsilon_{t}$ which we then extend to $u_{t}^{*}$.

Lemma A.3 establishes some moments for this series, while Lemma A.4 establishes the corresponding invariance principle. Lemma A.5 then extends this to $u_{t}^{*}$.

Lemma A.3. Define $H_{m}^{*}=b^{-1 / 2} \sum_{s=1}^{b}\left(\varepsilon_{i_{m}+s}-E^{*} \varepsilon_{i_{m}+s}\right)$. If Assumptions 1 and 3 hold, we have

(i) $\mathrm{E}^{*} H_{m}^{*}=0$,

(ii) $\mathrm{E}^{*} H_{m}^{*} H_{m}^{* \prime}=\Sigma+o_{p}(1)$.

Proof of Lemma A.2. Statement (i) is trivial. To prove statement (ii), write

$$
\begin{aligned}
\mathrm{E}^{*} H_{m}^{*} H_{m}^{* \prime}= & b^{-1} \sum_{s_{1}=1}^{b} \sum_{s_{2}=1}^{b}\left(\mathrm{E}^{*} \varepsilon_{i_{m}+s_{1}} \varepsilon_{i_{m}+s_{2}}^{\prime}-\mathrm{E}^{*} \varepsilon_{i_{m}+s_{1}} \mathrm{E}^{*} \varepsilon_{i_{m}+s_{2}}^{\prime}\right) \\
= & \frac{1}{b(T-b)} \sum_{s_{1}=1}^{b} \sum_{s_{2}=1}^{b} \sum_{t=1}^{T-b} \varepsilon_{t+s_{1}} \varepsilon_{t+s_{2}}^{\prime} \\
& -b^{-1}\left(\frac{1}{T-b} \sum_{s=1}^{b} \sum_{t=1}^{T-b} \varepsilon_{t+s}\right)\left(\frac{1}{T-b} \sum_{s=1}^{b} \sum_{t=1}^{T-b} \varepsilon_{t+s}\right)^{\prime} \\
= & A_{T}+B_{T} .
\end{aligned}
$$


Let us first look at $B_{T}$. Note that

$$
\begin{aligned}
& \frac{1}{T-b} \sum_{s=1}^{b} \sum_{t=1}^{T-b} \varepsilon_{t+s}=\frac{b}{T} \sum_{t=1}^{T} \varepsilon_{t}+\frac{b^{2}}{T(T-b)} \sum_{t=1}^{T} \varepsilon_{t} \\
& \quad-\frac{1}{T-b} \sum_{s=1}^{b} \sum_{t=1}^{s-1} \varepsilon_{t}-\frac{1}{T-b} \sum_{s=1}^{b} \sum_{t=T-b+s+1}^{T} \varepsilon_{t} \\
& =O_{p}\left(b T^{-1 / 2}\right)+O_{p}\left(b^{2} T^{-3 / 2}\right)+O_{p}\left(b^{3 / 2} T^{-1}\right)+O_{p}\left(b^{3 / 2} T^{-1}\right),
\end{aligned}
$$

from which we can conclude that $B_{T}=O_{p}\left(b T^{-1}\right)$.

Next we look at the first term. We have

$$
\begin{aligned}
A_{T}= & \frac{1}{b(T-b)} \sum_{s=1}^{b} \sum_{t=1}^{T-b} \varepsilon_{t+s} \varepsilon_{t+s}^{\prime} \\
& +\frac{1}{b(T-b)} \sum_{s_{1}=1}^{b} \sum_{s_{2}=1, s_{1} \neq s_{2}}^{b} \sum_{t=1}^{T-b} \varepsilon_{t+s_{1}} \varepsilon_{t+s_{2}}^{\prime} \\
= & T^{-1} \sum_{t=1}^{T} \varepsilon_{t} \varepsilon_{t}^{\prime}+O_{p}\left(b T^{-1 / 2}\right)=\Sigma+o_{p}(1) .
\end{aligned}
$$

This concludes the proof of part (ii).

Lemma A.4. Let Assumptions 1 and 3 hold. Then, as $T \rightarrow \infty$,

$$
\begin{aligned}
W_{T}^{*}(r) & =T^{-1 / 2} \sum_{k=0}^{\lfloor(k-1) r\rfloor} \sum_{s=1}^{b}\left(\varepsilon_{i_{m}+s}-\mathrm{E}^{*} \varepsilon_{i_{m}+s}\right) \\
& \stackrel{d^{*}}{\rightarrow} \Sigma^{1 / 2} W(r) \text { in probability. }
\end{aligned}
$$

Proof of Lemma A.4. First note that

$T^{-1 / 2} \sum_{m=0}^{\lfloor(k-1) r\rfloor} \sum_{s=1}^{b}\left(\varepsilon_{i_{m}+s}-\mathrm{E}^{*} \varepsilon_{i_{m}+s}\right)=k^{-1 / 2} \sum_{m=0}^{\lfloor(k-1) r\rfloor} H_{m}^{*}$.

It is easily seen that the conditions of Corollary 2.2 of Phillips and Durlauf (1986) hold for the $H_{m}^{*}$ terms, by which the result follows.

Lemma A.5. Let $y_{t}$ be generated under $H_{0}$ setting (A). Let Assumptions 1 and 3 hold. Then, as $T \rightarrow \infty$,

$T^{-1 / 2} \sum_{m=0}^{\lfloor(k-1) r\rfloor} \sum_{s=1}^{b}\left(u_{i_{m}+s}-\mathrm{E}^{*} u_{i_{m}+s}\right) \stackrel{d^{*}}{\rightarrow} \Gamma^{\prime} \Psi(1) \Sigma^{1 / 2} W(r)$.

\section{Proof of Lemma A.5. As}

$$
\begin{aligned}
& T^{-1 / 2} \sum_{m=0}^{\lfloor(k-1) r\rfloor} \sum_{s=1}^{b}\left(u_{i_{m}+s}-\mathrm{E}^{*} u_{i_{m}+s}\right) \\
& =T^{-1 / 2} \sum_{m=0}^{\lfloor(k-1) r\rfloor} \sum_{s=1}^{b}\left(\Gamma^{\prime} x_{i_{m}+s}-\mathrm{E}^{*} \Gamma^{\prime} x_{i_{m}+s}\right) \\
& =\Gamma^{\prime}\left(T^{-1 / 2} \sum_{m=0}^{\lfloor(k-1) r\rfloor} \sum_{s=1}^{b}\left(x_{i_{m}+s}-\mathrm{E}^{*} x_{i_{m}+s}\right)\right),
\end{aligned}
$$

we focus on $T^{-1 / 2} \sum_{m=0}^{\lfloor(k-1) r\rfloor} \sum_{s=1}^{b}\left(x_{i_{m}+s}-\mathrm{E}^{*} x_{i_{m}+s}\right)$.
Using the Beveridge-Nelson decomposition we have

$$
\begin{aligned}
T^{-1 / 2} & \sum_{m=0}^{\lfloor(k-1) r\rfloor} \sum_{s=1}^{b}\left(x_{i_{m}+s}-\mathrm{E}^{*} x_{i_{m}+s}\right) \\
= & T^{-1 / 2} \sum_{m=0}^{\lfloor(k-1) r\rfloor} \sum_{s=1}^{b} \Psi(1)\left(\varepsilon_{i_{m}+s}-\mathrm{E}^{*} \varepsilon_{i_{m}+s}\right) \\
& -T^{-1 / 2} \sum_{m=0}^{L(k-1) r\rfloor}\left(\left(\tilde{\Psi}(L) \varepsilon_{i_{m}+b}-\mathrm{E}^{*} \tilde{\Psi}(L) \varepsilon_{i_{m}+b}\right)\right. \\
& \left.\times\left(\tilde{\Psi}(L) \varepsilon_{i_{m}}-\mathrm{E}^{*} \tilde{\Psi}(L) \varepsilon_{i_{m}}\right)\right),
\end{aligned}
$$

where $\tilde{\Psi}(z)=\sum_{j=0}^{\infty} \tilde{\Psi}_{j} z^{j}, \tilde{\Psi}_{j}=\sum_{i=j+1}^{\infty} \Psi_{j}$.

We will show that $T^{-1 / 2} \sum_{m=0}^{\lfloor(k-1) r\rfloor}\left(\tilde{\Psi}(L) \varepsilon_{i_{m}+b}-\mathrm{E}^{*} \tilde{\Psi}(L) \varepsilon_{i_{m}+b}\right)$ $=o_{p}^{*}(1)$. First note that

$$
\begin{aligned}
\mathrm{P}^{*} & {\left[\left|T^{-1 / 2} \sum_{m=0}^{\lfloor(k-1) r\rfloor}\left(\tilde{\Psi}(L) \varepsilon_{i_{m}+s}-\mathrm{E}^{*} \tilde{\Psi}(L) \varepsilon_{i_{m}+s}\right)\right|>\epsilon\right] } \\
& \leq \frac{1}{\epsilon^{2}} \mathrm{E}^{*}\left|T^{-1 / 2} \sum_{m=0}^{\lfloor(k-1) r\rfloor}\left(\tilde{\Psi}(L) \varepsilon_{i_{m}+s}-\mathrm{E}^{*} \tilde{\Psi}(L) \varepsilon_{i_{m}+s}\right)\right|^{2} \\
& =\frac{1}{\epsilon^{2}} E^{*}\left|G_{T, s}^{*}\right|^{2}
\end{aligned}
$$

for $s=0, b$ by the Markov inequality. Then, letting $\xi_{t}^{*}=\varepsilon_{t}-\mathrm{E}^{*} \varepsilon_{t}$,

$$
\begin{aligned}
\mathrm{E}^{*}\left|G_{T, s}^{*}\right|^{2}= & T^{-1} \sum_{m_{1}=0}^{\lfloor(k-1) r\rfloor} \sum_{m_{2}=0}^{\lfloor(k-1) r\rfloor} \mathrm{E}^{*}\left(\sum_{j=0}^{\infty} \tilde{\Psi}_{j} \xi_{i_{m_{1}}+s-j}^{*}\right)^{\prime} \\
& \times\left(\sum_{j=0}^{\infty} \tilde{\Psi}_{j} \xi_{i_{m_{2}}+s-j}^{*}\right) \\
= & T^{-1} \sum_{m=0}^{\lfloor(k-1) r\rfloor} \mathrm{E}^{*}\left|\sum_{j=0}^{\infty} \tilde{\Psi}_{j}\left(\varepsilon_{i_{m}+s-j}-\mathrm{E}^{*} \varepsilon_{i_{m}+s-j}\right)\right|^{2},
\end{aligned}
$$

using the independence of the blocks. Now, by Minkowski's inequality, we have uniformly in $r$,

$$
\begin{aligned}
& \mathrm{E}^{*}\left|G_{T, s}^{*}\right|^{2} \\
& \leq T^{-1} \sum_{m=0}^{\lfloor(k-1) r\rfloor}\left[\sum_{j=0}^{\infty}\left|\tilde{\Psi}_{j}\right|\left(\mathrm{E}^{*}\left|\varepsilon_{i_{m}+s-j}-\mathrm{E}^{*} \varepsilon_{i_{m}+s-j}\right|^{2}\right)^{1 / 2}\right]^{2} \\
& \quad \leq 4 k T^{-1}\left(\sum_{j=0}^{\infty}\left|\tilde{\Psi}_{j}\right|\right)^{2} \max _{j} \frac{1}{T-b} \sum_{t=1}^{T-b}\left|\varepsilon_{t+s-j}\right|^{2}
\end{aligned}
$$

A sufficient condition for $\sum_{j=0}^{\infty}\left|\tilde{\Psi}_{j}\right|<\infty$ is that $\sum_{j=0}^{\infty} j\left|\Psi_{j}\right|<$ $\infty$; see Phillips and Solo (1992, Lemma 2.1). This holds by Assumption 1. We also have that

$\frac{1}{T-b} \sum_{t=1}^{T-b}\left|\varepsilon_{t+s-j}\right|=O_{p}(1)$

by the moment conditions in Assumption 1. Therefore $\mathrm{E}^{*}\left|G_{T, s}^{*}\right|^{2}=$ $O_{p}\left(b^{-1}\right)$ for $s=0, b$ from which it follows that, uniformly in $r$,

$T^{-1 / 2} \sum_{m=0}^{\lfloor(k-1) r\rfloor} \sum_{s=1}^{b}\left(x_{i_{m}+s}-\mathrm{E}^{*} x_{i_{m}+s}\right)=\Psi(1) W_{T}^{*}(r)+o_{p}^{*}(1)$

and therefore 


$$
\begin{aligned}
& T^{-1 / 2} \sum_{m=0}^{\lfloor(k-1) r\rfloor} \sum_{s=1}^{b}\left(x_{i_{m}+s}-\mathrm{E}^{*} x_{i_{m}+s}\right) \\
& \stackrel{d^{*}}{\rightarrow} \Psi(1) \Sigma^{1 / 2} W(r) \quad \text { in probability }
\end{aligned}
$$

by Lemma A.4. The proof is concluded by referring to (29) and applying the continuous mapping theorem.

Proof of Lemma 2. Note that

$S_{T}^{*}(r)=T^{-1 / 2} y_{1}+T^{-1 / 2} \sum_{m=0}^{M_{r}-1} \sum_{s=1}^{b} \hat{u}_{i_{m}+s}+T^{-1 / 2} \sum_{s=1}^{N_{r}} \hat{u}_{i_{M_{r}}+s}$,

where $M_{r}=\lfloor(\lfloor T r\rfloor-2) / b\rfloor$ and $N_{r}=\lfloor T r\rfloor-M_{r} b-1$. As $T^{-1 / 2} y_{1}=O_{p}\left(T^{-1 / 2}\right)$, we write

$S_{T}^{*}(r)=T^{-1 / 2} \sum_{m=0}^{M_{r}} \sum_{s=1}^{b} \hat{u}_{i_{m}+s}-T^{-1 / 2} \sum_{s=N_{r}+1}^{b} \hat{u}_{i_{M_{r}}+s}+O_{p}\left(T^{-1 / 2}\right)$.

It now follows from applying the proof of Theorem 3.1 of Paparoditis and Politis (2003) to each element of $S_{T}^{*}(r)$ that, uniformly in $r$,

$S_{T}^{*}(r)=T^{-1 / 2} \sum_{m=0}^{\lfloor(k-1) r\rfloor} \sum_{s=1}^{b}\left(u_{i_{m}+s}-E^{*} u_{i_{m}+s}\right)+o_{p}^{*}(1)$.

The proof is then concluded by applying Lemma A.5.

The next step is to determine the moments of the bootstrap series corresponding to the moments in Lemma A.1.

Lemma A.6. Let $y_{t}$ be generated under $H_{0}$ setting (A). Let Assumptions 1 and 3 hold. Then, as $T \rightarrow \infty$,

$$
\begin{aligned}
\text { (i) } \Omega^{*}=T^{-1}\left[\mathrm{E}^{*}\left(\sum_{t=1}^{T} u_{t}^{*}\right)\left(\sum_{t=1}^{T} u_{t}^{*}\right)^{\prime}-\mathrm{E}^{*}\left(\sum_{t=1}^{T} u_{t}^{*}\right)\right. \\
\\
\left.\mathrm{E}^{*}\left(\sum_{t=1}^{T} u_{t}^{*}\right)^{\prime}\right]=\Gamma^{\prime} \Psi(1) \Sigma \Psi(1)^{\prime} \Gamma+o_{p}(1), \\
\text { (ii) } \Omega_{0}^{*}=T^{-1} \sum_{t=1}^{T}\left[\mathrm{E}^{*}\left(u_{t}^{*} u_{t}^{* \prime}\right)-\mathrm{E}^{*} u_{t}^{*} \mathrm{E}^{*} u_{t}^{* \prime}\right] \\
=\sum_{i=0}^{\infty} \Gamma^{\prime} \Psi_{i} \Sigma \Psi_{i}^{\prime} \Gamma+o_{p}(1) .
\end{aligned}
$$

Proof of Lemma A.6. We start with part (i). Using the arguments in the proof of Lemma 2 (take $r=1$ ) we can show that

$$
T^{-1 / 2} \sum_{t=1}^{T} u_{t}^{*}=T^{-1 / 2} \sum_{m=0}^{k-1} \sum_{s=1}^{b}\left(u_{i_{m}+s}-\mathrm{E}^{*} u_{i_{m}+s}\right)+o_{p}^{*}(1) .
$$

Therefore $E^{*}\left[T^{-1 / 2} \sum_{t=1}^{T} u_{t}^{*}\right]=o_{p}(1)$ and

$$
\begin{aligned}
\Omega^{*}= & T^{-1} \mathrm{E}^{*}\left(\sum_{m=0}^{k-1} \sum_{s=1}^{b}\left(u_{i_{m}+s}-\mathrm{E}^{*} u_{i_{m}+s}\right)\right) \\
& \times\left(\sum_{m=0}^{k-1} \sum_{s=1}^{b}\left(u_{i_{m}+s}-\mathrm{E}^{*} u_{i_{m}+s}\right)\right)^{\prime}+o_{p}(1) .
\end{aligned}
$$

Using the Beveridge-Nelson decomposition, we can show, as in the proof of Lemma A.5, that

$$
\begin{aligned}
& T^{-1 / 2} \sum_{m=0}^{k-1} \sum_{s=1}^{b}\left(u_{i_{m}+s}-\mathrm{E}^{*} u_{i_{m}+s}\right) \\
& =T^{-1 / 2} \Gamma^{\prime} \Psi(1) \sum_{m=0}^{k-1} \sum_{s=1}^{b}\left(\varepsilon_{i_{m}+s}-\mathrm{E}^{*} \varepsilon_{i_{m}+s}\right)+o_{p}^{*}(1) .
\end{aligned}
$$

Consequently

$$
\begin{aligned}
\Omega^{*}= & T^{-1} \Gamma^{\prime} \Psi(1) \mathrm{E}^{*}\left[\left(\sum_{m=0}^{k-1} \sum_{s=1}^{b}\left(\varepsilon_{i_{m}+s}-\mathrm{E}^{*} \varepsilon_{i_{m}+s}\right)\right)\right. \\
& \left.\times\left(\sum_{m=0}^{k-1} \sum_{s=1}^{b}\left(\varepsilon_{i_{m}+s}-\mathrm{E}^{*} \varepsilon_{i_{m}+s}\right)\right)^{\prime}\right] \Psi(1)^{\prime} \Gamma+o_{p}(1) \\
= & \Gamma^{\prime} \Psi(1) \Sigma \Psi(1)^{\prime} \Gamma+o_{p}(1),
\end{aligned}
$$

which follows from the independence of the blocks and Lemma A.3. This concludes the proof of part (i).

The proof of part (ii) is similar to part (i). As in the proof of Lemma 2 we have that

$$
\begin{aligned}
\Omega_{0}= & T^{-1} \sum_{m=0}^{k-1} \sum_{s=1}^{b} \mathrm{E}^{*}\left(u_{i_{m}+s}-\mathrm{E}^{*} u_{i_{m}+s}\right)\left(u_{i_{m}+s}-\mathrm{E}^{*} u_{i_{m}+s}\right)^{\prime} \\
& +o_{p}(1) .
\end{aligned}
$$

Then, using that $u_{i_{m}+s}=\sum_{j=0}^{\infty} \Gamma^{\prime} \Psi_{j} \varepsilon_{i_{m}+s-j}$, we can write

$$
\begin{aligned}
\Omega_{0}^{*}= & T^{-1} \sum_{m=0}^{k-1} \sum_{s=1}^{b} \mathrm{E}^{*}\left(\sum_{j=0}^{\infty} \Gamma^{\prime} \Psi_{j}\left(\varepsilon_{i_{m}+s-j}-\mathrm{E}^{*} \varepsilon_{i_{m}+s-j}\right)\right) \\
& \times\left(\sum_{j=0}^{\infty} \Gamma^{\prime} \Psi_{j}\left(\varepsilon_{i_{m}+s-j}-\mathrm{E}^{*} \varepsilon_{i_{m}+s-j}\right)\right)^{\prime}+o_{p}(1) \\
= & T^{-1} \sum_{m=0}^{k-1} \sum_{s=1}^{b} \sum_{i=0}^{\infty} \sum_{j=0}^{\infty} \Gamma^{\prime} \Psi_{i} \frac{1}{T-b} \\
& \times \sum_{t=1}^{T-b}\left(\varepsilon_{t+s-i} \varepsilon_{t+s-j}^{\prime}\right) \Psi_{j}^{\prime} \Gamma \\
& -T^{-1} \sum_{m=0}^{k-1} \sum_{s=1}^{b} \sum_{i=0}^{\infty} \sum_{j=0}^{\infty} \Gamma^{\prime} \Psi_{i} \frac{1}{T-b} \\
& \times \sum_{t=1}^{T-b} \varepsilon_{t+s-i} \frac{1}{T-b} \sum_{t=1}^{T-b} \varepsilon_{t+s-j}^{\prime} \Psi_{j}^{\prime} \Gamma+o_{p}(1) \\
= & A_{T}+B_{T}+o_{p}(1) .
\end{aligned}
$$

Note that

$$
\begin{aligned}
\left|B_{T}\right| \leq & b \max _{1 \leq s \leq b}\left|\frac{1}{T-b} \sum_{t=1}^{T-b} \varepsilon_{t+s}\right|^{2}|\Gamma|^{2} \\
& \times \sum_{i=0}^{\infty} \sum_{j=0}^{\infty}\left|\Psi_{i}\right|\left|\Psi_{j}\right|=b O_{p}\left(T^{-1}\right) O(1)=O_{p}\left(k^{-1}\right),
\end{aligned}
$$

while

$A_{T}=\frac{1}{(T-b) b} \sum_{t=1}^{T-b} \sum_{s=1}^{b} \sum_{i=0}^{\infty} \Gamma^{\prime} \Psi_{i}\left(\varepsilon_{t+s-i} \varepsilon_{t+s-i}^{\prime}\right) \Psi_{i}^{\prime} \Gamma+o_{p}(1)$,

and as $\frac{1}{(T-b) b} \sum_{t=1}^{T-b} \sum_{s=1}^{b} \varepsilon_{t+s-i} \varepsilon_{t+s-i}^{\prime}=\Sigma+o_{p}(1)$, we can conclude that $\Omega_{0}^{*}=\sum_{i=0}^{\infty} \Gamma^{\prime} \Psi_{i} \Sigma \Psi_{i}^{\prime} \Gamma+o_{p}(1)$.

Lemma A.7. Let $y_{t}$ be generated under $H_{0}$ setting (A). Let Assumptions 1 and 3 hold. Then, as $T \rightarrow \infty$, we have for $i=1, \ldots, N$,

(i) $T^{-1} \sum_{t=1}^{T} y_{i, t-1}^{*} \Delta y_{i, t}^{*} \stackrel{d}{\rightarrow} \int_{0}^{1} B_{i}(r) \mathrm{d} B_{i}(r)+\frac{1}{2}\left(\omega_{i}-\omega_{0, i}\right)$,

(ii) $T^{-2} \sum_{t=1}^{T} y_{i, t-1}^{* 2} \stackrel{d}{\rightarrow} \int_{0}^{1} B_{i}(r)^{2} \mathrm{~d} r$,

where convergence also holds jointly.

Proof of Lemma A.7. The result follows from Lemmas 2 and A.6 and the continuous mapping theorem. Joint convergence can again be established using the Cramér-Wold device. 
Proof of Theorem 2. The result follows directly from Lemma A.7.

Proof of Lemma 3. As in Lemma 1, we have by Assumption 1 that $W_{T}(r)=T^{-1 / 2} \sum_{t=1}^{\lfloor T r\rfloor} \varepsilon_{t} \stackrel{d}{\rightarrow} \Sigma^{1 / 2} W(r)$. Then it follows that $T^{-1 / 2} \sum_{t=1}^{\lfloor T r\rfloor} f_{t}=\Psi_{2}(1) W_{T}(r)+o_{p}(1)$ uniformly in $r$, and consequently $T^{-1 / 2} \sum_{t=1}^{\lfloor T r\rfloor} f_{t} \stackrel{d}{\rightarrow} \Psi_{2}(1) \Sigma^{1 / 2} W(r)$.

Now

$$
\begin{aligned}
T^{-1 / 2} \sum_{t=1}^{\lfloor T r\rfloor} u_{t} & =T^{-1 / 2} \sum_{t=1}^{\lfloor T r\rfloor} \Lambda f_{t}+T^{-1 / 2} \sum_{t=1}^{\lfloor T r\rfloor} \Delta w_{t} \\
& =T^{-1 / 2} \sum_{t=1}^{\lfloor T r\rfloor} \Lambda f_{t}+T^{-1 / 2}\left(w_{\lfloor T r\rfloor}-w_{0}\right) \\
& =T^{-1 / 2} \sum_{t=1}^{\lfloor T r\rfloor} \Lambda f_{t}+O_{p}\left(T^{-1 / 2}\right)
\end{aligned}
$$

uniformly in $r$ and $T^{-1 / 2} \sum_{t=1}^{\lfloor T r} \Lambda f_{t} \stackrel{d}{\rightarrow} \Lambda \Psi_{2}(1) \Sigma^{1 / 2} W(r)$.

The next lemma is the counterpart of Lemma A.1.

Lemma A.8. Let $y_{t}$ be generated under $H_{0}$ setting (B). Let Assumptions 1 and 2 hold. Then

(i) $\bar{\Omega}=\lim _{T \rightarrow \infty} T^{-1} \mathrm{E}\left(\sum_{t=1}^{T} u_{t}\right)\left(\sum_{t=1}^{T} u_{t}\right)^{\prime}$

$=\Lambda \Psi_{2}(1) \Sigma \Psi_{2}(1)^{\prime} \Lambda^{\prime}$

(ii) $\bar{\Omega}_{0}=\lim _{T \rightarrow \infty} T^{-1} \sum_{t=1}^{T} \mathrm{E}\left(u_{t} u_{t}^{\prime}\right)=\sum_{j=0}^{\infty}\left(\Lambda \Psi_{2, j} \Sigma \Psi_{2, j}^{\prime} \Lambda^{\prime}+\right.$ $\left(\bar{\Psi}_{1, j}-\bar{\Psi}_{1, j+1}\right) \Sigma \Psi_{2, j}^{\prime} \Lambda^{\prime}+\Lambda \Psi_{2, j} \Sigma\left(\bar{\Psi}_{1, j}-\bar{\Psi}_{1, j+1}\right)^{\prime}+2 \bar{\Psi}_{1, j} \Sigma \bar{\Psi}_{1, j}^{\prime}-$ $\left.\bar{\Psi}_{1, j} \Sigma \bar{\Psi}_{1, j+1}^{\prime}-\bar{\Psi}_{1, j+1} \Sigma \bar{\Psi}_{1, j}^{\prime}\right)$.

Proof of Lemma A.8. For part (i), note that

$$
\begin{aligned}
\bar{\Omega} & =\lim _{T \rightarrow \infty} T^{-1} \mathrm{E}\left(\sum_{t=1}^{T} \Lambda f_{t}+w_{T}-w_{0}\right)\left(\sum_{t=1}^{T} \Lambda f_{t}+w_{T}-w_{0}\right)^{\prime} \\
& =\lim _{T \rightarrow \infty} T^{-1} \sum_{s=1}^{T} \sum_{t=1}^{T} \Lambda \mathrm{E}\left(f_{s} f_{t}^{\prime}\right) \Lambda^{\prime}=\Lambda \Psi_{2}(1) \Sigma \Psi_{2}(1)^{\prime} \Lambda^{\prime},
\end{aligned}
$$

where the last step follows as in the proof of Lemma A.1 part (i). For part (ii) we have

$$
\begin{aligned}
\bar{\Omega}_{0}= & \lim _{T \rightarrow \infty} T^{-1} \sum_{t=1}^{T} \mathrm{E}\left(\left(\Lambda f_{t}+\Delta w_{t}\right)\left(\Lambda f_{t}+\Delta w_{t}\right)^{\prime}\right) \\
= & \lim _{T \rightarrow \infty}\left\{T^{-1} \sum_{t=1}^{T} \Lambda \mathrm{E}\left(f_{t} f_{t}^{\prime}\right) \Lambda^{\prime}+T^{-1} \sum_{t=1}^{T} \mathrm{E}\left(\Delta w_{t} f_{t}^{\prime}\right) \Lambda^{\prime}\right. \\
& \left.+T^{-1} \sum_{t=1}^{T} \Lambda \mathrm{E}\left(f_{t} \Delta w_{t}^{\prime}\right)+T^{-1} \sum_{t=1}^{T} \mathrm{E}\left(\Delta w_{t} \Delta w_{t}^{\prime}\right)\right\} \\
= & A_{T}+B_{T}+B_{T}^{\prime}+C_{T} .
\end{aligned}
$$

Now

$A_{T}=\sum_{j=0}^{\infty} \Lambda \Psi_{2, j} \Sigma \Psi_{2, j}^{\prime} \Lambda^{\prime}$,

analogous to the proof of Lemma A.1 part (ii). Similarly

$B_{T}=\sum_{j=0}^{\infty}\left(\bar{\Psi}_{1, j}-\bar{\Psi}_{1, j+1}\right) \Sigma \Psi_{2, j}^{\prime} \Lambda^{\prime}$

and

$C_{T}=\sum_{j=0}^{\infty}\left(2 \bar{\Psi}_{1, j} \Sigma \bar{\Psi}_{1, j}^{\prime}-\bar{\Psi}_{1, j} \Sigma \bar{\Psi}_{1, j+1}^{\prime}-\bar{\Psi}_{1, j+1} \Sigma \bar{\Psi}_{1, j}^{\prime}\right)$.

This completes the proof.
Proof of Theorem 3. Using Lemmas 3, A.8 and the continuous mapping theorem we can construct the counterpart of Lemma A.2. The result then follows.

Lemma A.9. Let $y_{t}$ be generated under $H_{0}$ setting (B). Let Assumptions 1-3 hold. Then, as $T \rightarrow \infty$,

$T^{-1 / 2} \sum_{m=0}^{\lfloor(k-1) r\rfloor} \sum_{s=1}^{b}\left(u_{i_{m}+s}-E^{*} u_{i_{m}+s}\right) \stackrel{d^{*}}{\rightarrow} \Lambda \Psi_{2}(1) \Sigma^{1 / 2} W(r)$.

Proof of Lemma A.9. Note that

$$
\begin{aligned}
T^{-1 / 2} & \sum_{m=0}^{\lfloor(k-1) r\rfloor} \sum_{s=1}^{b}\left(u_{i_{m}+s}-\mathrm{E}^{*} u_{i_{m}+s}\right) \\
= & \Lambda\left(T^{-1 / 2} \sum_{m=0}^{\lfloor(k-1) r\rfloor} \sum_{s=1}^{b}\left(f_{i_{m}+s}-\mathrm{E}^{*} f_{i_{m}+s}\right)\right)+T^{-1 / 2} \\
& \times \sum_{m=0}^{\lfloor(k-1) r\rfloor}\left(w_{i_{m}+b}-\mathrm{E}^{*} w_{i_{m}+b}\right)-T^{-1 / 2} \sum_{m=0}^{\lfloor(k-1) r\rfloor}\left(w_{i_{m}}-\mathrm{E}^{*} w_{i_{m}}\right) \\
= & A_{T}^{*}-B_{T, 0}^{*}+B_{T, b}^{*} .
\end{aligned}
$$

We want to show that $B_{T, s}^{*}=O_{p}^{*}\left(b^{-1 / 2}\right)$ uniformly in $r$ for $s=0, b$. First note that by Eq. (30)

$B_{T, s}^{*}=T^{-1 / 2} \sum_{m=0}^{\lfloor(k-1) r\rfloor}\left(\bar{\Psi}_{1}(L) \varepsilon_{i_{m}+s}-\mathrm{E}^{*} \bar{\Psi}_{1}(L) \varepsilon_{i_{m}+s}\right)+o_{p}^{*}(1)$.

As $k^{-1 / 2} \sum_{m=0}^{\lfloor(k-1) r\rfloor}\left(\bar{\Psi}_{1}(L) \varepsilon_{i_{m}+s}-E^{*} \bar{\Psi}_{1}(L) \varepsilon_{i_{m}+s}\right)=O_{p}^{*}(1)$, it follows that $B_{T, s}^{*}=O_{p}^{*}\left(b^{-1 / 2}\right)$ uniformly in $r$ for $s=0, b$.

As in the proof of Lemma A.5 we have that

$T^{-1 / 2} \sum_{m=0}^{\lfloor(k-1) r\rfloor} \sum_{s=1}^{b}\left(f_{i_{m}+s}-\mathrm{E}^{*} f_{i_{m}+s}\right)=\Psi_{2}(1) W_{T}^{*}(r)+o_{p}^{*}(1)$

uniformly in $r$ and consequently that

$$
\begin{gathered}
T^{-1 / 2} \sum_{m=0}^{\lfloor(k-1) r\rfloor} \sum_{s=1}^{b}\left(u_{i_{m}+s}-E^{*} u_{i_{m}+s}\right) \\
\stackrel{d^{*}}{\rightarrow} \Lambda \Psi_{2}(1) W(r) \quad \text { in probability. }
\end{gathered}
$$

Proof of Lemma 4. As the order of the $f_{t}$ determines the order of $u_{t}$, the proof proceeds as the proof of Lemma 2 such that we have, uniformly in $r$,

$S_{T}^{*}(r)=T^{-1 / 2} \sum_{m=0}^{\lfloor(k-1) r\rfloor} \sum_{s=1}^{b}\left(u_{i, i_{m}+s}-\mathrm{E}^{*} u_{i, i_{m}+s}\right)+o_{p}^{*}(1)$.

The proof is then concluded by applying Lemma A.9.

We consider the bootstrap moments in the following lemma.

Lemma A.10. Let $y_{t}$ be generated under $H_{0}$ setting (B). Let Assumptions 1-3 hold. Then, as $T \rightarrow \infty$,

(i) $\bar{\Omega}^{*}=T^{-1} \mathrm{E}\left(\sum_{t=1}^{T} u_{t}^{*}\right)\left(\sum_{t=1}^{T} u_{t}^{*}\right)^{\prime}=\Lambda \Psi_{2}(1) \Sigma \Psi_{2}(1)^{\prime} \Lambda^{\prime}+$ $o_{p}(1)$,

(ii) $\bar{\Omega}_{0}^{*}=T^{-1} \sum_{t=1}^{T} \mathrm{E}\left(u_{t}^{*} u_{t}^{* \prime}\right)=\sum_{j=0}^{\infty}\left(\Lambda \Psi_{2, j} \Sigma \Psi_{2, j}^{\prime} \Lambda^{\prime}+\left(\bar{\Psi}_{1, j}-\right.\right.$ $\left.\bar{\Psi}_{1, j+1}\right) \Sigma \Psi_{2, j}^{\prime} \Lambda^{\prime}+\Lambda \Psi_{2, j} \Sigma\left(\bar{\Psi}_{1, j}-\bar{\Psi}_{1, j+1}\right)^{\prime}+2 \bar{\Psi}_{1, j} \Sigma \bar{\Psi}_{1, j}^{\prime}-$ $\left.\bar{\Psi}_{1, j} \Sigma \bar{\Psi}_{1, j+1}^{\prime}-\bar{\Psi}_{1, j+1} \Sigma \bar{\Psi}_{1, j}^{\prime}\right)+o_{p}(1)$. 
Proof of Lemma A.10. We start with part (i). As

$T^{-1 / 2} \sum_{t=1}^{T} u_{t}^{*}=T^{-1 / 2} \sum_{m=0}^{k-1} \sum_{s=1}^{b}\left(u_{i_{m}+s}-\mathrm{E}^{*} u_{i_{m}+s}\right)+o_{p}^{*}(1)$,

we have that

$$
\begin{aligned}
\bar{\Omega}^{*}= & T^{-1} \mathrm{E}^{*}\left(\sum_{m=0}^{k-1} \sum_{s=1}^{b}\left(u_{i_{m}+s}-\mathrm{E}^{*} u_{i_{m}+s}\right)\right) \\
& \times\left(\sum_{m=0}^{k-1} \sum_{s=1}^{b}\left(u_{i_{m}+s}-\mathrm{E}^{*} u_{i_{m}+s}\right)\right)^{\prime}+o_{p}(1) .
\end{aligned}
$$

Combining the proof of Lemmas A.5 and A.9 we can show that

$$
\begin{aligned}
& T^{-1 / 2} \sum_{m=0}^{k-1} \sum_{s=1}^{b}\left(u_{i_{m}+s}-\mathrm{E}^{*} u_{i_{m}+s}\right) \\
& =T^{-1 / 2} \Lambda \Psi_{2}(1) \sum_{m=0}^{k-1} \sum_{s=1}^{b}\left(\varepsilon_{i_{m}+s}-\mathrm{E}^{*} \varepsilon_{i_{m}+s}\right)+o_{p}^{*}(1) .
\end{aligned}
$$

Consequently

$\bar{\Omega}^{*}=\Lambda \Psi_{2}(1) \Sigma \Psi_{2}(1)^{\prime} \Lambda^{\prime}+o_{p}(1)$,

which follows as in the proof of Lemma A.6. This concludes the proof of part (i).

Next we consider part (ii). As in the proof of Lemma A.6 we can show that

$$
\begin{aligned}
\bar{\Omega}_{0}^{*}= & T^{-1} \sum_{m=0}^{k-1} \sum_{s=1}^{b} E^{*}\left(u_{i_{m}+s}-E^{*} u_{i_{m}+s}\right)\left(u_{i_{m}+s}-E^{*} u_{i_{m}+s}\right)^{\prime} \\
& +o_{p}(1) .
\end{aligned}
$$

Then we can write

$$
\begin{aligned}
\bar{\Omega}_{0}^{*}= & T^{-1} \sum_{m=0}^{k-1} \sum_{s=1}^{b} \Lambda \mathrm{E}^{*}\left(f_{i_{m}+s-j}-\mathrm{E}^{*} f_{i_{m}+s-j}\right) \\
& \times\left(f_{i_{m}+s-j}-\mathrm{E}^{*} f_{i_{m}+s-j}\right)^{\prime} \Lambda^{\prime} \\
& +T^{-1} \sum_{m=0}^{k-1} \sum_{s=1}^{b} \Lambda \mathrm{E}^{*}\left(f_{i_{m}+s-j}-\mathrm{E}^{*} f_{i_{m}+s-j}\right) \\
& \times\left(\Delta w_{i_{m}+s-j}-\mathrm{E}^{*} \Delta w_{i_{m}+s-j}\right)^{\prime} \\
& +T^{-1} \sum_{m=0}^{k-1} \sum_{s=1}^{b} \mathrm{E}^{*}\left(\Delta w_{i_{m}+s-j}-\mathrm{E}^{*} \Delta w_{i_{m}+s-j}\right) \\
& \times\left(f_{i_{m}+s-j}-\mathrm{E}^{*} f_{i_{m}+s-j}\right)^{\prime} \Lambda^{\prime} \\
& +T^{-1} \sum_{m=0}^{k-1} \sum_{s=1}^{b} \mathrm{E}^{*}\left(\Delta w_{i_{m}+s-j}-\mathrm{E}^{*} \Delta w_{i_{m}+s-j}\right) \\
& \times\left(\Delta w_{i_{m}+s-j}-\mathrm{E}^{*} \Delta w_{i_{m}+s-j}\right)^{\prime} \\
= & A_{T}^{*}+B_{T}^{*}+B_{T}^{* \prime}+C_{T}^{*} .
\end{aligned}
$$

Then we can show, in the same way as in the proof of Lemma A.6 part (ii), that

$A_{T}^{*}=\sum_{j=0}^{\infty} \Lambda \Psi_{2, j} \Sigma \Psi_{2, j}^{\prime} \Lambda^{\prime}+o_{p}(1)$,

as well as

$B_{T}^{*}=\sum_{j=0}^{\infty}\left(\bar{\Psi}_{1, j}-\bar{\Psi}_{1, j+1}\right) \Sigma \Psi_{2, j}^{\prime} \Lambda^{\prime}+o_{p}(1)$ and

$C_{T}^{*}=\sum_{j=0}^{\infty}\left(2 \bar{\Psi}_{1, j} \Sigma \bar{\Psi}_{1, j}^{\prime}-\bar{\Psi}_{1, j} \Sigma \bar{\Psi}_{1, j+1}^{\prime}-\bar{\Psi}_{1, j+1} \Sigma \bar{\Psi}_{1, j}^{\prime}\right)+o_{p}(1)$.

This completes the proof.

Proof of Theorem 4. As for Theorem 3 we can construct the counterpart of Lemma A.7 using Lemmas 4, A.10 and the continuous mapping theorem. The result then follows.

Proof of Lemma 5. We can write

$T^{-1} \tau_{p}=\frac{\sum_{i=1}^{N} T^{-1} \sum_{t=2}^{T} y_{i, t-1} \Delta y_{i, t}}{\sum_{i=1}^{N} T^{-1} \sum_{t=2}^{T} y_{i, t-1}^{2}}=\frac{\sum_{i=1}^{N} a_{i T}}{\sum_{i=1}^{N} b_{i T}}$.

Now as $y_{i, t}$ is a stationary process for all $i=1, \ldots, N$, we have that $a_{i T}=T^{-1} \sum_{t=2}^{T} y_{i, t-1} y_{i, t}-T^{-1} \sum_{t=2}^{T} y_{i, t-1}^{2} \stackrel{p}{\rightarrow} \gamma_{i}(1)-\gamma_{i}(0)$,

and

$b_{i T}=T^{-1} \sum_{t=2}^{T} y_{i, t-1}^{2} \stackrel{p}{\rightarrow} \gamma_{i}(0)$.

Similarly,

$T^{-1} \tau_{g m}=N^{-1} \sum_{i=1}^{N} \frac{a_{i T}}{b_{i T}}$,

from which the result follows.

Lemma A.11. Let $y_{t}$ be generated under $H_{1}^{a}$. Let Assumptions 1 and 3 hold. Then, as $T \rightarrow \infty$,

$T^{-1 / 2} \sum_{m=0}^{\lfloor(k-1) r\rfloor} \sum_{s=1}^{b}\left(u_{i_{m}+s}-\mathrm{E}^{*} u_{i_{m}+s}\right) \stackrel{d^{*}}{\rightarrow} \Psi^{++}(1) \Sigma^{1 / 2} W(r)$.

Proof of Lemma A.11. Using the Beveridge-Nelson decomposition we can write

$$
\begin{aligned}
T^{-1 / 2} & \sum_{m=0}^{\lfloor(k-1) r\rfloor} \sum_{s=1}^{b}\left(u_{i_{m}+s}-\mathrm{E}^{*} u_{i_{m}+s}\right) \\
= & T^{-1 / 2} \sum_{m=0}^{\lfloor(k-1) r\rfloor} \sum_{s=1}^{b} \Psi^{++}(1)\left(\varepsilon_{i_{m}+s}-\mathrm{E}^{*} \varepsilon_{i_{m}+s}\right) \\
& -T^{-1 / 2} \sum_{m=0}^{\lfloor(k-1) r\rfloor}\left(\left(\tilde{\Psi}^{++}(L) \varepsilon_{i_{m}+b}-\mathrm{E}^{*} \tilde{\Psi}^{++}(L) \varepsilon_{i_{m}+b}\right)\right. \\
& \left.\times\left(\tilde{\Psi}^{++}(L) \varepsilon_{i_{m}}-\mathrm{E}^{*} \tilde{\Psi}(L) \varepsilon_{i_{m}}\right)\right),
\end{aligned}
$$

where $\tilde{\Psi}^{++}(z)=\sum_{j=0}^{\infty} \tilde{\Psi}_{j}^{++} z^{j}$ and $\tilde{\Psi}_{j}^{++}=\sum_{i=j+1}^{\infty} \Psi_{j}^{++}$.

We need to show that $T^{-1 / 2} \sum_{m=0}^{\lfloor(k-1) r\rfloor}\left(\tilde{\Psi}^{++}(L) \varepsilon_{i_{m}+b}-E^{*} \tilde{\Psi}(L)^{++}\right.$ $\left.\varepsilon_{i_{m}+b}\right)=o_{p}^{*}(1)$, uniformly in $r$. Completely analogous to the proof of Lemma A.5 this means showing that $\sum_{j=0}^{\infty}\left|\tilde{\Psi}_{j}^{++}\right|<\infty$ or equivalently $\sum_{j=0}^{\infty} j\left|\Psi_{j}^{++}\right|<\infty$. This holds as we remarked that the summability condition continues to hold.

Proof of Theorem 5. We can apply the proof of Theorem 3.1 of Paparoditis and Politis (2003), now for the stationary case, to show that, uniformly in $r$.

$S_{T}^{*}(r)=T^{-1 / 2} \sum_{m=0}^{\lfloor(k-1) r\rfloor} \sum_{s=1}^{b}\left(u_{i, i_{m}+s}-\mathrm{E}^{*} u_{i, i_{m}+s}\right)+o_{p}^{*}(1)$. 
The result now follows by applying Lemma A.11 and the continuous mapping theorem.

\section{Proof of Lemma 6. We write}

$$
\begin{aligned}
\tau_{p}=\frac{\sum_{i=1}^{N} T^{-1} \sum_{t=2}^{T} y_{i, t-1} \Delta y_{i, t}}{\sum_{i=1}^{N} T^{-2} \sum_{t=2}^{T} y_{i, t-1}^{2}} \\
=\frac{\sum_{i=1}^{n_{1}} T^{-1} \sum_{t=2}^{T} y_{i, t-1} \Delta y_{i, t}+\sum_{i=n_{1}+1}^{N} T^{-1} \sum_{t=2}^{T} y_{i, t-1} \Delta y_{i, t}}{T^{-1} \sum_{i=1}^{n_{1}} T^{-1} \sum_{t=2}^{T} y_{i, t-1}^{2}+\sum_{i=n_{1}+1}^{N} T^{-2} \sum_{t=2}^{T} y_{i, t-1}^{2}} \\
=\frac{\sum_{i=1}^{n_{1}} a_{i T}+\sum_{i=n_{1}+1}^{N} c_{i T}}{\sum_{i=1}^{n_{1}} T^{-1} b_{i T}+\sum_{i=n_{1}+1}^{N} d_{i T}} .
\end{aligned}
$$

The convergence of $a_{i T}$ and $b_{i T}$ follow from the proof of Lemma 5. Furthermore, as in Lemma A.2, we have that

$c_{i T} \stackrel{d}{\rightarrow} \int_{0}^{1} B_{i}^{\#}(r) \mathrm{d} B_{i}^{\#}(r)$,

$d_{i T} \stackrel{d}{\rightarrow} \int_{0}^{1} B_{i}^{\#}(r)^{2} \mathrm{~d} r$

from which the result for $\tau_{p}$ follows.

For $\tau_{g m}$ we can write

$$
\begin{aligned}
T^{-1} \tau_{g m} & =N^{-1} \sum_{i=1}^{n_{1}} \frac{a_{i T}}{b_{i T}}+N^{-1} \sum_{i=n_{1}+1}^{N} T^{-1} \frac{c_{i T}}{d_{i T}} \\
& =N^{-1} \sum_{i=1}^{n_{1}} \frac{a_{i T}}{b_{i T}}+O_{p}\left(T^{-1}\right) . \quad
\end{aligned}
$$

Proof of Corollary 1. The proof is immediate by combining the proofs of Theorems 2 and 5.

\section{References}

Bai, J., Ng, S., 2004. A PANIC attack on unit roots and cointegration. Econometrica 72, 1127-1177.

Bai, J., Ng, S., 2010. Panel unit root tests with cross-section dependence: a further investigation. Econometric Theory 26, 1088-1114.

Breitung, J., Das, S., 2008. Testing for unit roots in panels with a factor structure. Econometric Theory 24, 88-108.

Bühlmann, P., Künsch, H.R., 1999. Block length selection in the bootstrap for time series. Computational Statistics \& Data Analysis 31, 295-310.

Chang, Y., 2004. Bootstrap unit root tests in panels with cross-sectional dependency. Journal of Econometrics 120, 263-293.

Chang, Y., Song, W., 2009. Testing for unit roots in small panels with short-run and long-run cross-sectional dependencies. Review of Economic Studies 76, 903-935.
Choi, I., Chue, T.K., 2007. Subsampling hypothesis tests for nonstationary panels with applications to exchange rates and stock prices. Journal of Applied Econometrics 22, 401-428.

Chudik, A., Pesaran, M.H., Tosetti, E., 2009. Weak and strong cross section dependence and estimation of large panels. Working Paper Series No. 1100. European Central Bank.

Davidson, J., 2000. Econometric Theory, 2nd ed. Blackwell Publishers, Oxford.

Davidson, J., 2002. Stochastic Limit Theory, 2nd ed. Oxford University Press, Oxford

Di Iorio, F., Fachin, S., 2008. Testing for cointegration in dependent panels via residual-based bootstrap methods. Working Paper.

Fachin, S., 2007. Long-run trends in internal migrations in Italy: a study in panel cointegration with dependent units. Journal of Applied Econometrics 22, 401-428.

Gengenbach, C., Palm, F.C., Urbain, J.-P., 2010. Panel unit root tests in the presence of cross-sectional dependencies: comparison and implications for modelling. Econometric Reviews 29, 111-145.

Giacomini, R., Politis, D.N., White, H., 2007. A warp-speed method for conducting Monte Carlo experiments involving bootstrap estimators. Working Paper.

Gonçalves, S., Vogelsang, T.J., 2010. Block bootstrap puzzles in HAC robust testing: the sophistication of the naive bootstrap. Econometric Theory (forthcoming).

Hall, P., Horowitz, J.L., Jing, B.-Y., 1995. On blocking rules for the bootstrap with dependent data. Biometrika 82, 561-574.

Härdle, W., Horowitz, J.L., Kreiss, J.-P., 2003. Bootstrap methods for time series International Statistical Review 71, 435-459.

Hlouskova, J., Wagner, M., 2006. The performance of panel unit root and stationarity tests: results from a large scale simulation study. Econometric Reviews 25 85-116.

Horowitz, J.L., Savin, N.E., 2000. Empirically relevant critical values for hypothesis tests: a bootstrap approach. Journal of Econometrics 95, 375-389.

Im, K.S., Pesaran, M.H., Shin, Y., 2003. Testing for unit roots in heterogeneous panels. Journal of Econometrics 115, 53-74.

Kapetanios, G., 2008. A bootstrap procedure for panel data sets with many crosssectional units. Econometrics Journal 11, 377-395.

Künsch, H.R., 1989. The jackknife and the bootstrap for general stationary observations. Annals of Statistics 17, 1217-1241.

Lahiri, S.N., 2003. Resampling Methods for Dependent Data. Springer-Verlag, New York.

Lahiri, S.N., Furukawa, K., Lee, Y.-D., 2007. A nonparametric plug-in rule for selecting optimal block lengths for block bootstrap methods. Statistical Methodology 4 292-321.

Levin, A., Lin, C.-F., Chu, C.-S.J., 2002. Unit root tests in panel data: asymptotic and finite-sample properties. Journal of Econometrics 108, 1-24.

Maddala, G.S., Wu, S., 1999. A comparative study for unit root tests with panel data and a simple new test. Oxford Bulletin of Economics and Statistics 61 631-652.

Moon, H.R., Perron, B., 2004. Testing for a unit root in panels with dynamic factors. Journal of Econometrics 122, 81-126.

Moon, H.R., Perron, B., Phillips, P.C.B., 2007. Incidental trends and the power of panel unit root tests. Journal of Econometrics 141, 416-459.

O'Connell, P.G.J., 1998. The overvaluation of purchasing power parity. Journal of International Economics 44, 1-19.

Paparoditis, E., Politis, D.N., 2003. Residual-based block bootstrap for unit root testing. Econometrica 71, 813-855.

Pedroni, P., Vogelsang, T.J., Wagner, M., Westerlund, J., 2008. Robust unit root and cointegration rank tests for time series panels. Working Paper.

Pesaran, M.H., 2007. A simple panel unit root test in the presence of cross-sectional dependence. Journal of Applied Econometrics 22, 265-312.

Phillips, P.C.B., Durlauf, S.N., 1986. Multiple time series regression with integrated processes. Review of Economic Studies 53, 473-495.

Phillips, P.C.B., Solo, V., 1992. Asymptotics for linear processes. Annals of Statistics 20, 971-1001.

Politis, D.N., Romano, J.P., Wolf, M., 1999. Subsampling. Springer-Verlag, New York

Politis, D.N., White, H., 2004. Automatic block-length selection for the dependent bootstrap. Econometric Reviews 23, 53-70.

Smeekes, S., 2009. Detrending bootstrap unit root tests. METEOR Research Memorandum 09/056. Maastricht University. 